Preprint typeset in JHEP style - HYPER VERSION

UH-511-1190-12

\title{
Natural Supersymmetry: LHC, dark matter and ILC searches
}

\author{
Howard Baer $^{a}$, Vernon Barger ${ }^{b}$, Peisi Huang ${ }^{b}$ and Xerxes Tata ${ }^{c}$ \\ ${ }^{a}$ Dept. of Physics and Astronomy, University of Oklahoma, Norman, OK 73019, USA \\ ${ }^{b}$ Dept. of Physics, University of Wisconsin, Madison, WI 53706, USA \\ ${ }^{c}$ Dept. of Physics and Astronomy, University of Hawaii, Honolulu, HI 96822, USA \\ E-mail: baer@nhn.ou.edu, barger@pheno.wisc.edu, phuang7@wisc.edu, \\ tata@phys.hawaii.edu
}

ABSTRACT: Particle physics models with Natural Supersymmetry are characterized by a superpotential parameter $\mu \sim m_{h} \sim 125 \mathrm{GeV}$, while third generation squarks have mass $\lesssim 0.5-1.5 \mathrm{TeV}$. Gluinos should be lighter than several $\mathrm{TeV}$ so as not to destabilize the lighter squarks. First and second generation sfermions can be at the tens-of-TeV level which yields a decoupling solution to the SUSY flavor and $C P$ problems. Adopting a top-down approach, we delineate the range of GUT scale SUSY model parameters which leads to a Natural SUSY mass spectrum. We find natural SUSY models to be tightly constrained by the $b \rightarrow s \gamma$ branching fraction measurement while it is also difficult but not impossible to accommodate a light Higgs scalar of mass $\simeq 125 \mathrm{GeV}$. We present several benchmark points which are expandable to slopes and planes. Natural SUSY is difficult to see at LHC unless some third generation squarks are very light. The top- and bottomsquarks cascade decay mainly to higgsino-like charginos and neutralinos via numerous posibilities, leading to a rather complex set of signatures. Meanwhile, a linear $e^{+} e^{-}$collider operating at $\sqrt{s} \sim 0.25-0.5 \mathrm{TeV}$ would be a higgsino factory and is essentially guaranteed a SUSY discovery of the low-lying charged and neutral higgsino states. Since thermal neutralino cold dark matter is underproduced, we conjecture that the incorporation of a Peccei-Quinn sector or light moduli into the theory will augment higgsino dark matter production, possibly together with an admixture of axions. We present rates for direct and indirect higgsino dark matter detection for the case where light higgsinos dominate the dark matter abundance.

Keywords: Supersymmetry Phenomenology, Supersymmetric Standard Model, Large Hadron Collider. 


\section{Introduction}

\subsection{Impact of LHC sparticle searches}

The search for weak scale supersymmetry (SUSY) [1, 2, 3] has begun in earnest at the CERN Large Hadron Collider (LHC). From a non-observation of multi-jet plus multilepton $+E_{T}^{\text {miss }}$ events with or without tagged $b$-jets in a data sample of $\sim 1-4.5 \mathrm{fb}^{-1}$, the CMS [4] and ATLAS [5] experiments have excluded gluinos and squarks up to $1.4 \mathrm{TeV}$ for $m_{\tilde{g}} \simeq m_{\tilde{q}}$ and gluinos up to $\sim 0.8 \mathrm{TeV}$, for the case of $m_{\tilde{q}} \gg m_{\tilde{g}}[6]$.

Many experimental analyses have been performed within the framework of the mSUGRA (or CMSSM) model, which assumes a common mass parameter $m_{0}$ (renormalized at the GUT scale) for all scalars, and likewise a common mass parameter $m_{1 / 2}$ for the gauginos. The physical spectrum- obtained by renormalization group (RG) running of soft mass parameters from $M_{\mathrm{GUT}}$ to $M_{\text {weak }}$ - is characterized by a squark mass spectrum with $m_{\tilde{q}} \sim m_{\tilde{g}}$ for low $m_{0} \lesssim m_{1 / 2}$ or $m_{\tilde{q}} \gg m_{\tilde{g}}$ for large values of $m_{0}$. Despite the fact that superpotential Yukawa interactions reduce third generation squark masses relative to those of first/second generation squarks, third generation squarks nonetheless frequently have masses $\gtrsim 1 \mathrm{TeV}$, putting them in conflict with electroweak fine-tuning constraints (discussed below). This has led some physicists to question whether weak scale SUSY indeed stabilizes the gauge hierarchy, given the constraints from the LHC. ${ }^{1}$

We emphasize that while the various squarks end up being nearly degenerate within the mSUGRA model, the limit on $m_{\tilde{q}}$ quoted above arises mainly from the production and decay of first-generation squarks. Only these squarks can be pair produced from the valence $u$ and $d$ quark content of the colliding protons. As a result, their production cross section falls off much less rapidly with increasing squark mass than the corresponding cross section for the production of second and third generation squarks: thus, the ATLAS and CMS limits, $m_{\tilde{q}} \gtrsim 1 \mathrm{TeV}$, apply to first generation squarks, while second and third generation squarks may be much lighter without being in conflict with either LHC data or with the notion of superpartners as the new physics that stabilizes the weak scale.

\subsection{Impact of LHC Higgs searches}

Recent results from LHC Higgs searches find tantalizing hints for a Standard Model (SM)like Higgs boson of mass $m_{h} \simeq 125 \mathrm{GeV}$ (although at present values of $m_{h} \sim 120 \mathrm{GeV}$ are also possible). Such a large value of $m_{h}$ is difficult to realize in models such as minimal anomaly mediation (mAMSB) or minimal gauge mediation (mGMSB) [7, 8] unless all sparticle masses are in the 10-20 TeV range, in severe conflict with electroweak fine-tuning constraints. Meanwhile, gravity mediation (SUGRA) remains a possible venue for communication of SUSY breaking since, unlike in mAMSB and mGMSB models, the scalar trilinear soft SUSY breaking coupling $A_{0}$ is an independent parameter, and can be chosen to be large as seems to be required by such large values of $m_{h}[$ [ $]$.

\footnotetext{
${ }^{1}$ By adjusting the trilinear soft breaking parameter $A_{0}$ to certain values, the $\tilde{t}_{1}$ mass may be dialed to sub-TeV values. However, the remaining third generation squark masses typically remain at large values and still in possible conflict with fine-tuning constraints.
} 
SUSY models based on gravity-mediation are, however, expected generically to give rise to large FCNC and CP-violating processes 10 since there is no mechanism to enforce the required generational universality [1, 2, 3] or alignment of fermion and sfermion mass matrices needed to reduce flavour-changing processes to an acceptable level. ${ }^{2}$ Indeed, the SUSY flavor and CP problem endemic to gravity-mediation has served as motivation for the construction of AMSB and GMSB models, since SUSY sources of FCNC and $C P$-violation are automatically suppressed in these models.

An alternative solution to the SUSY flavor and $C P$ problems arises by decoupling: allowing for first and second generation squark and slepton masses to be in the 10-50 TeV range. Third generation squark masses, which directly enter into electroweak fine-tuning, or "naturalness" considerations (see below), may be much lighter since flavor and $C P$ constraints are relatively mild for third generation particles 11. Supersymmetric models containing a split spectrum - sub-TeV third generation squarks but with multi-TeV first/second generation squarks - have been advocated for some time under the label of effective SUSY [12], or ESUSY 13]. Indeed, the non-observation of squarks and gluinos in the LHC data sample could be a hint pointing in this direction.

\subsection{Naturalness constraints}

It is well known [2] that at tree-level the magnitude of the higgsino mass parameter $\mu$ is determined in terms of (1) the weak scale soft SUSY breaking (SSB) mass parameters $m_{H_{u}}^{2}$ and $m_{H_{d}}^{2}$ that appear in the Higgs sector scalar potential, (2) the ratio $\tan \beta \equiv \frac{v_{u}}{v_{d}}$, and (3) the observed value of the $Z$-boson mass. Including radiative corrections via the effective potential method, this relation gets modified to:

$$
\frac{1}{2} M_{Z}^{2}=\frac{\left(m_{H_{d}}^{2}+\Sigma_{d}\right)-\left(m_{H_{u}}^{2}+\Sigma_{u}\right) \tan ^{2} \beta}{\left(\tan ^{2} \beta-1\right)}-\mu^{2} .
$$

Here, $\Sigma_{u}$ and $\Sigma_{d}$ arise from radiative corrections[14], and are given in the 1-loop approximation to the Higgs effective potential by

$$
\Sigma_{u, d}=\frac{1}{v_{u, d}} \frac{\partial \Delta V}{\partial H_{u, d}}
$$

where $\Delta V$ is the one-loop correction to the tree-level potential, and the derivative is evaluated in the physical vaccuum: i.e. the fields are set to their vacuum expectation values after evaluating the derivative.

It is reasonable to say that the theory naturally yields the correct value of $M_{Z}$ if the individual terms on the right hand side of Eq. (1.1) are comparable in magnitude so that the observed value of $M_{Z}$ is obtained without resorting to large cancellations. Indeed this is why $|\mu|$ has been suggested as a measure of naturalness[15], with theories where $\mu^{2} \lesssim M_{Z}^{2}$ being the "most natural". This relationship must be accepted with some latitude, since values of $\mu^{2} \lesssim(100 \mathrm{GeV})^{2}$ are phenomenologically excluded by the LEP2 limit that

\footnotetext{
${ }^{2}$ In mSUGRA, the SUSY GIM mechanism is imposed by simply assuming universality of scalar masses at the high scale, usually taken to be $M_{\mathrm{GUT}}$.
} 
$m_{\widetilde{W}_{1}}>103.5 \mathrm{GeV}$. Of course, there is nothing special about $\mu^{2}$ and the same considerations apply equally to all the terms, including those involving the radiative corrections.

In the following, we will somewhat arbitrarily require that each individual term in (1.1) is bounded by about $(200 \mathrm{GeV})^{2}$. Similar considerations have recently been adopted by several other groups 16, 17, 18, 19, 20. ${ }^{3}$ In distinction with other works, our focus is on the expected sparticle mass spectra and collider and dark matter phenomenology of Natural SUSY models with parameters defined at a high scale (taken to be $M_{G U T}$ ) which lead to weak scale parameters that are natural in the sense that we have just described.

The largest contributions to $\Sigma_{u, d}$ in Eq. (1.1) arise from superpotential Yukawa interactions of third generation squarks involving the top quark Yukawa coupling. The dominant contribution to these quantities is given by

$$
\Sigma_{u} \sim \frac{3 f_{t}^{2}}{16 \pi^{2}} \times m_{\tilde{t}_{i}}^{2}\left(\ln \left(m_{\tilde{t}_{i}^{2}} / Q^{2}\right)-1\right),
$$

and so grows quadratically with the top squark masses. Clearly, the top squark (and by $S U(2)$ gauge symmetry, also $\left.\tilde{b}_{L}\right)$ masses must then be bounded above by the naturalness conditions. In Ref. [20], it has been shown that requiring $\Sigma_{u} \lesssim \frac{1}{2} M_{Z}^{2}$ leads to $m_{\tilde{t}_{i}} \lesssim$ $500 \mathrm{GeV}$. Scaling this to allow $\mu$ values up to $150 \mathrm{GeV}(200 \mathrm{GeV})$ leads to a corresponding bound $m_{\tilde{t}_{i}} \lesssim 1 \mathrm{TeV}(1.5 \mathrm{TeV})$, which of course also applies to the heavier top squark. ${ }^{4}$ In other words, from this perspective, models with $\mu \lesssim 150-200 \mathrm{GeV}$ and top squarks at the $\mathrm{TeV}$ scale or below are completely natural. In this connection, it is perhaps worth remarking that since

$$
m_{A}^{2} \simeq 2 \mu^{2}+m_{H_{u}}^{2}+m_{H_{d}}^{2}+\Sigma_{u}+\Sigma_{d},
$$

for moderate to large values of $\tan \beta$, the heavier Higgs scalars can naturally be at the several-TeV scale because of the appearance of $\tan ^{2} \beta-1$ in the denominator of Eq. (1.1). Notice, however, that the bound of $(200 \mathrm{GeV})^{2}$ on each term in Eq. (1.1) translates to an upper bound

$$
m_{A} \sim\left|m_{H_{d}}^{2}\right|^{\frac{1}{2}} \lesssim|\mu| \tan \beta
$$

Such a constraint could prove theoretically significant in considerations of high scale models with special properties such as models with unified Yukawa couplings at the GUT scale 21.

Our discussion up to this point shows that SUSY models with $|\mu| \lesssim 150-200 \mathrm{GeV}$ and top squark masses (and if $\tan \beta$ is very large, also bottom squark masses) below 1-1.5 TeV are perfectly natural. There will also be corresponding constraints on other sparticles such as electro-weak charginos and neutralinos that also directly couple to the Higgs sector, but since these couplings are smaller than $f_{t}$ and because there are no colour factors, the constraints will be correspondingly weaker. Sparticles such as first and second generation squarks and sleptons that have no direct/significant couplings to the Higgs sector are

\footnotetext{
${ }^{3}$ These analyses differ in detail on the restrictions on each term, and even whether a common constraint is applied to each term. For this reason, some of the constraints that have been obtained by these analyses are stronger than the ones we obtain in this paper.

${ }^{4}$ For very large values of $\tan \beta$ where the bottom quark Yukawa becomes comparable to $f_{t}$, similar considerations would apply to bottom squarks.
} 
constrained only via two-loop effects and can easily be in the $10-50 \mathrm{TeV}$ range. An important exception would be the gluino, since radiative corrections to the top squark mass are proportional to $m_{\tilde{g}} 17$. Using $\delta m_{\tilde{q}}^{2} \sim \frac{2 g_{s}^{2}}{3 \pi^{2}} m_{\tilde{g}}^{2} \times \log$ and setting logs to be order

unity, we see that $m_{\tilde{g}} \lesssim 3 m_{\tilde{q}}$. For top squarks to remain below the $1.5 \mathrm{TeV}$ range, the gluino must be lighter than about $4 \mathrm{TeV}$. In models with electroweak gaugino mass unification, electroweak-inos would then automatically not destroy naturalness.

\subsection{Natural SUSY}

These considerations suggest that the region of SUSY parameter space where

- $|\mu| \lesssim 150-200 \mathrm{GeV}$,

- third generation squarks $m_{\tilde{t}_{L, R}}, m_{\tilde{b}_{L}} \lesssim 1-1.5 \mathrm{TeV}$,

- $m_{\tilde{g}} \lesssim 3-4 \mathrm{TeV}$ and SSB electroweak-ino masses smaller than 1-2 TeV

- $m_{A} \lesssim|\mu| \tan \beta$,

- $m_{\tilde{q}_{1,2}}, m_{\tilde{\ell}_{1,2}} \sim 10-50 \mathrm{TeV}$,

may, from naturalness and flavor $/ C P$ considerations, merit a dedicated study. The first and second generation squarks and sleptons - lying in the 10-50 $\mathrm{TeV}$ range - provide a decoupling solution to the SUSY flavour problem, the SUSY CP problem and to the problem of too-rapid-proton decay. We remark here that if SUSY breaking arises from supergravity breaking in a hidden sector, then the gravitino mass $m_{3 / 2}$ sets the scale for the largest of the SSB terms, and we would also expect $m_{3 / 2} \sim 10-50 \mathrm{TeV}$ : such a high value of $m_{3 / 2}$ also provides a solution to the gravitino problem 22, 23]. The heavier Higgs bosons may easily be in the several-TeV range for moderate to large values of $\tan \beta$.

SUSY models with the above generic spectra have been dubbed "Natural SUSY" 18 and are a more restrictive case of effective SUSY models because we further restrict $|\mu| \lesssim$ $150-200 \mathrm{GeV}$. This usually gives rise to a higgsino-like lightest neutralino $\widetilde{Z}_{1}$.

\subsection{Dark matter in natural SUSY}

In fact, a problem with effective SUSY models with a bino-like $\widetilde{Z}_{1}$ arises in that a vast overabundance of neutralino cold dark matter $(\mathrm{CDM})$ is expected[13], typically 2-4 orders of magnitude above the WMAP-measured value of $\Omega_{C D M} h^{2} \sim 0.11$ unless weak scale parameters happen to be in special parameter space regions. It has been suggested in 13. that if the strong CP problem is solved by the Peccei-Quinn (PQ) mechanism - which introduces a supermultiplet containing spin-zero axion and saxion fields, along with a spin- $\frac{1}{2}$ axino - then neutralinos might decay to a light axino LSP via $\widetilde{Z}_{1} \rightarrow \tilde{a} \gamma$. Since each neutralino converts to one axino, the decay-produced axino abundance is given by $\Omega_{\tilde{a}}^{N T P}=m_{\tilde{a}} / m_{\widetilde{Z}_{1}} \Omega_{\widetilde{Z}_{1}} h^{2}$. For $m_{\tilde{a}}$ in the $\mathrm{MeV}$ range, the suppression factor is $\sim 10^{-3}-10^{-5}$, bringing the DM abundance into accord with measurement.

However, typically in gravity mediation models the axino mass is expected to be around the TeV scale[24, 25], with $\widetilde{Z}_{1}$ remaining as LSP. In fact, in the PQ-augmented SUSY 
model, one then expects thermal axino production (TP) in the early universe, followed by late-time $\tilde{a} \rightarrow \widetilde{Z}_{1} \gamma$ decays, so the dark matter overabundance is made even worse. In the case of natural SUSY, the higgsino-like $\widetilde{Z}_{1}$ leads typically to a thermal underabundance of neutralino CDM. But now TP axinos followed by their decay to neutralinos can augment this abundance 26, 27, 28], while any remaining underabundance can be filled by axions produced via vacuum mis-alignment (coherent oscillations). Thus, in this case we might expect the CDM to consist of a higgsino-axion admixture. Which of these two particles dominates the DM abundance depends on specific choices of $\mathrm{PQ}$ parameters and on the value of re-heating temperature $T_{R}$ after inflation.

\section{Parameter space and mass spectra for Natural SUSY}

Since the introduction of softly broken SUSY into the Standard Model (leading to the Minimal Supersymmetric Standard Model, or MSSM) leads to a theory with stable mass hierarchies, it is natural to assume the MSSM is the low energy effective theory arising from an underlying SUSY Grand Unified Theory (GUT) which is broken at some high energy scale, taken here for definiteness to be $M_{G U T} \simeq 2 \times 10^{16} \mathrm{GeV}$. Indeed, the MSSM (or MSSM plus gauge singlets and/or additional complete $S U(5)$ multiplets) receives some indirect support from experiment in that 1 . the measured weak scale gauge couplings unify nearly to a point at $M_{G U T}$ under MSSM renormalization group (RG) evolution and 2. the MSSM electroweak symmetry is broken radiatively due to the large top quark Yukawa coupling, consistent with the measured value of $m_{t}$.

Motivated by these SUSY success stories, the interesting question arises as to whether the natural SUSY sparticle mass spectrum can be consistently generated from a model with parameters defined at the high scale $Q=M_{G U T}$. To implement a low value of $|\mu| \lesssim$ $150-200 \mathrm{GeV}$, we will adopt the 2-parameter non-universal Higgs model (NUHM2) [29], wherein weak scale values of $\mu$ and $m_{A}$ may be used as inputs in lieu of GUT scale values of $m_{H_{u}}^{2}$ and $m_{H_{d}}^{2}$. To generate the split first/second versus third generation scalar mass hierarchy, we will adopt a common GUT scale mass $m_{0}(3)$ for third generation scalars, and a common GUT scale mass $m_{0}(1,2)$ for the first/second generation scalars. The intra-generational mass universality is well-motivated by $S O(10)$ GUT symmetry, since all matter multiplets of a single generation live in the 16-dimensional spinor rep of $S O(10)$. We can also allow some degree of non-universality between $m_{0}(1)$ and $m_{0}(2)$ so long as both lie in the tens of $\mathrm{TeV}$ regime, and provide a decoupling solutions to SUSY FCNC and $C P$-violating processes (for constraints from FCNC processes, see Ref. [30]). For convenience, we will take them as degenerate.

To allow for a light third generation, we adopt different GUT scale values for the scalar mass parameter of the first two generations and the third generation. In the spirit of SUSY GUT theories, we will assume gaugino mass unification to a common gaugino mass $m_{1 / 2}$, and assume a universal trilinear scalar coupling $A_{0}$ at the GUT scale. The sparticle mass spectrum together with sparticle couplings is then determined by the parameter set, ${ }^{5}$

$$
m_{0}(1,2), m_{0}(3), m_{1 / 2}, A_{0}, \tan \beta, \mu, m_{A} .
$$

\footnotetext{
${ }^{5}$ We could have allowed a $D$-term contribution that would arise when the additional $U(1)$ that is in
} 
We take $m_{t}=173.3 \mathrm{GeV}$.

Our goal in this Section is to search for weak scale spectra that are natural in the sense defined above within this framework, to delineate regions of parameter space consistent with low energy constraints, and to study their implications for SUSY searches at the LHC.

For our calculations, we adopt the Isajet 7.82 [31] SUSY spectrum generator Isasugra 32]. Isasugra begins the calculation of the sparticle mass spectrum with input $\overline{D R}$ gauge couplings and $f_{b}, f_{\tau}$ Yukawa couplings at the scale $Q=M_{Z}\left(f_{t}\right.$ running begins at $\left.Q=m_{t}\right)$ and evolves the 6 couplings up in energy to scale $Q=M_{G U T}$ (defined as the value $Q$ where $\left.g_{1}=g_{2}\right)$ using two-loop RGEs. We do not strictly enforce the unification condition $g_{3}=g_{1}=g_{2}$ at $M_{G U T}$, since a few percent deviation from unification can be attributed to unknown GUT-scale threshold corrections [33]. At $Q=M_{G U T}$, we introduce the SSB parameters in (2.1) as boundary conditions, and evolve the set of 26 coupled MSSM RGEs 34] back down in scale to $Q=M_{Z}$. Full two-loop MSSM RGEs are used for soft term evolution, while the gauge and Yukawa coupling evolution includes threshold effects in the one-loop beta-functions, so the gauge and Yukawa couplings transition smoothly from the MSSM to SM effective theories as different mass thresholds are passed. In Isasugra, the values of SSB terms which mix are frozen out at the scale $Q \equiv M_{S U S Y}=\sqrt{m_{\tilde{t}_{L}} m_{\tilde{t}_{R}}}$, while non-mixing SSB terms are frozen out at their own mass scale [32]. The scalar potential is minimized using the RG-improved one-loop MSSM effective potential evaluated at an optimized scale $Q=M_{S U S Y}$ which accounts for leading two-loop effects [35]. Once the tree-level sparticle mass spectrum is computed, full one-loop radiative corrections are calculated for all sparticle and Higgs boson masses, including complete one-loop weak scale threshold corrections for the top, bottom and tau masses at scale $Q=M_{S U S Y}$ [36]. Since the GUT scale Yukawa couplings are modified by the threshold corrections, the Isajet RGE solution must be imposed iteratively with successive up-down running until a convergent sparticle mass solution is found. Since Isasugra uses a "tower of effective theories" approach to RG evolution, we expect a more accurate evaluation of the sparticle mass spectrum for models with split spectra (this procedure sums the logarithms of potentially large ratios of sparticle masses) than with programs which make an all-at-once transition from the MSSM to SM effective theories.

We searched for Natural SUSY solutions in the above parameter space by first fixing $\mu=150 \mathrm{GeV}$, and then performing a (linearly weighted) random scan over the remaining parameters in the following ranges:

$$
\begin{array}{r}
m_{0}(1,2): 5-50 \mathrm{TeV} \\
m_{0}(3): 0-5 \mathrm{TeV} \\
m_{1 / 2}: 0-5 \mathrm{TeV} \\
-4<A_{0} / m_{0}(3)<4
\end{array}
$$

$S O(10)$ but not in the $S U(5)$ subgroup is spontaneously broken. However, because we allow $\mu$ and $m_{A}$ as inputs, this would, however, have no impact on the allowed range of $m_{h}$, and so would only affect sparticle masses. In order to avoid a time-consuming scan over yet one more parameter which would probably not qualitatively alter the main results presented below, we have not included this term in our parametrization of the model. 


$$
\begin{array}{r}
m_{A}: \quad 0.15-2 \mathrm{TeV}, \\
\tan \beta: 1-60 .
\end{array}
$$

We require of our solutions that (1) electroweak symmetry be radiatively broken (REWSB), (2) the neutralino $\widetilde{Z}_{1}$ is the lightest MSSM particle, (3) the light chargino mass obeys the rather model independent LEP2 limit that $m_{\widetilde{W}_{1}}>103.5 \mathrm{GeV}$ [37] and (4) that $m_{\tilde{g}}<4$ $\mathrm{TeV}$, in accord with our naturalness criterion detailed above.

The results of our scan are plotted in Fig. 11. On the $y$-axis, we plot the average third generation squark mass

$$
\bar{m}_{\tilde{q}}(3)=\left(m_{\tilde{t}_{1}}+m_{\tilde{t}_{2}}+m_{\tilde{b}_{1}}\right) / 3
$$

while the $x$-axis lists the particular parameter. Blue points have $\bar{m}_{\tilde{q}}(3)<1.5 \mathrm{TeV}$, green points have $\bar{m}_{\tilde{q}}(3)<1 \mathrm{TeV}$ and red points have $\bar{m}_{\tilde{q}}(3)<0.5 \mathrm{TeV}$.

In frame $a$ ), we see that we can generate solutions with $\bar{m}_{\tilde{q}}(3)$ lower than $0.5 \mathrm{TeV}$, but only for values of $m_{0}(1,2) \lesssim 18 \mathrm{TeV}$. For heavier values of $m_{0}(1,2)$, it is well known that two-loop RGE effects tend to push third generation squark masses into the tachyonic range 38, 39, 13], which here would correspond to color breaking minima in the scalar potential. On the other hand, requiring $\bar{m}_{\tilde{q}}(3)<1$ (1.5) TeV allows for $m_{0}(1,2)$ as high as $\sim 25 \mathrm{TeV}$ - enough to suppress FCNCs except in the case of very large flavor violating soft terms 38]. In frame $b$ ), we plot the required value of $m_{0}(3)$ to give rise to sub-TeV average squark masses: here, values of $m_{0}(3)<2(5) \mathrm{TeV}$ are required to generate solutions with $\bar{m}_{\tilde{q}}(3)<0.5(1) \mathrm{TeV}$. Frame $c$ ) shows the value of $m_{1 / 2}$ required for natural SUSY models. A value of $m_{1 / 2}<1.4 \mathrm{TeV}$ is required for $\bar{m}_{\tilde{q}}(3)<0.5 \mathrm{TeV}$, while $m_{1.2} \lesssim 1.7 \mathrm{TeV}$ because we impose $m_{\tilde{g}} \lesssim 4 \mathrm{TeV}$. In frame $d$ ), we see that $\bar{m}_{\tilde{q}}(3)<0.5 \mathrm{TeV}$ can only be achieved for $A_{0} \gtrsim 0$, while $\bar{m}_{\tilde{q}}(3)<1 \mathrm{TeV}$ is allowed for $A_{0}>-2 m_{0}(3)$, i.e. $A_{0}$ cannot be large, negative. In frame $e$ ), we find that $\bar{m}_{\tilde{q}}(3)<0.5$ is allowed for $\tan \beta<50$, while $\bar{m}_{\tilde{q}}(3)<1$ $\mathrm{TeV}$ can be achieved for any $\tan \beta$ from $\sim 2-60$. Finally, frame $f$ ) shows that solutions with $\bar{m}_{\tilde{q}}(3)<0.5 \mathrm{TeV}$ can be found for any value of $m_{A}: 0.15-2 \mathrm{TeV}$.

In Fig. 2, we show the value of $m_{h}$ which is generated in NS models versus various SUSY parameters. In frame $a)$, we see that the red points with $\bar{m}_{\tilde{q}}(3)<0.5 \mathrm{TeV}$ populate the range $m_{h} \sim 105-120 \mathrm{GeV}$, while $m_{h}$ values, as obtained using Isajet, up to $123 \mathrm{GeV}$ $(124 \mathrm{GeV})$ can be readily accommodated for $\bar{m}_{\tilde{q}}(3)$ up to $1 \mathrm{TeV}(1.5 \mathrm{TeV})$. This should be compared with 115.5-131 GeV (114-127 GeV), the range of light Higgs boson masses currently allowed by the ATLAS (CMS) data 40, 41] at the 95\% CL. These experiments also report a small excess of a signal at $m_{h} \sim 125 \mathrm{GeV}$. For the smallest range of $\bar{m}_{\tilde{q}}(3)$ in the figure, it might appear that one would be hard pressed to accommodate the LHC hint of a 124-126 GeV light Higgs scalar. Of course, here one must keep in mind that Atlas/CMS may really be seeing a Higgs scalar with mass closer to $124 \mathrm{GeV}$ or that $m_{t}$ may be slightly larger than $173.2 \mathrm{GeV}$ as assumed in our calculation of the radiative correction. Combining this with a $\sim 3 \mathrm{GeV}$ error anticipated in the Isasugra calculation of $m_{h}$ and it becomes apparent that values of $m_{h} \sim 120-121 \mathrm{GeV}$ may be consistent with the Atlas/CMS $h(125)$ hint even for small values of $\bar{m}_{\tilde{q}}(3)$. The largest values of $m_{h}$ are obtained for $\tan \beta \gtrsim 10$. 

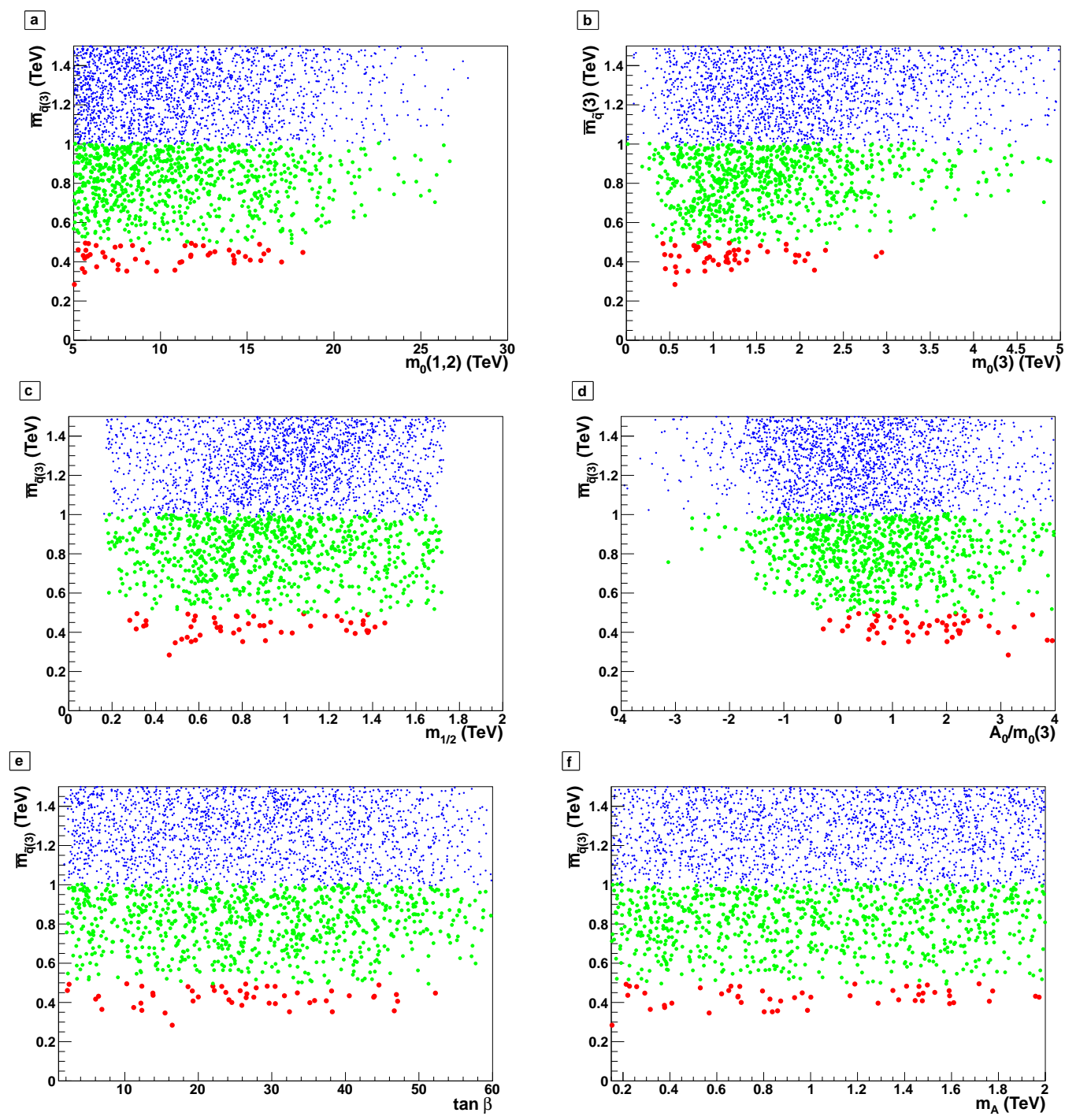

Figure 1: The value of $\bar{m}_{\tilde{q}}(3)$ versus various SUSY parameters with $\mu=150 \mathrm{GeV}$. The dots are colour-coded by the range of $\bar{m}_{\tilde{q}}(3): \leq 0.5 \mathrm{TeV}$ (red); $0.5-1 \mathrm{TeV}$ (green); $1-1.5 \mathrm{TeV}$ (blue).

We have already seen in Fig. 1 $1 d$ ) - and also here in Fig. 2 $d$ - that only $A_{0}>0$ values lead to $\bar{m}_{\tilde{q}}(3)<0.5 \mathrm{TeV}$, while in Ref. [9, 8] it is found that the largest values of $m_{h}$ are found for $A_{0} \sim-2 m_{0}$. As we allow increasing values of $\bar{m}_{\tilde{q}}(3)$ consistent with our naturalness conditions, we see that values of $A_{0} \sim-2 m_{0}(3)$ become allowed, and consequently higher values of $m_{h}$ can be accommodated. This is the case of maximal mixing in the top squark sector, which leads to maximal $m_{h}$ values 42.

The value of $B F(b \rightarrow s \gamma)$ should be rather tightly constraining for models of natural SUSY, since there may be several light third generation squarks, and not too heavy charginos, and since the main non-standard contributions to the decay rate come from top-squark-chargino loops 43. Here, we implement the Isatools subroutine IsaBSG 443] to compute the branching fraction, which is listed in Figure 3 versus $\bar{m}_{\tilde{q}}(3)$. These values are 

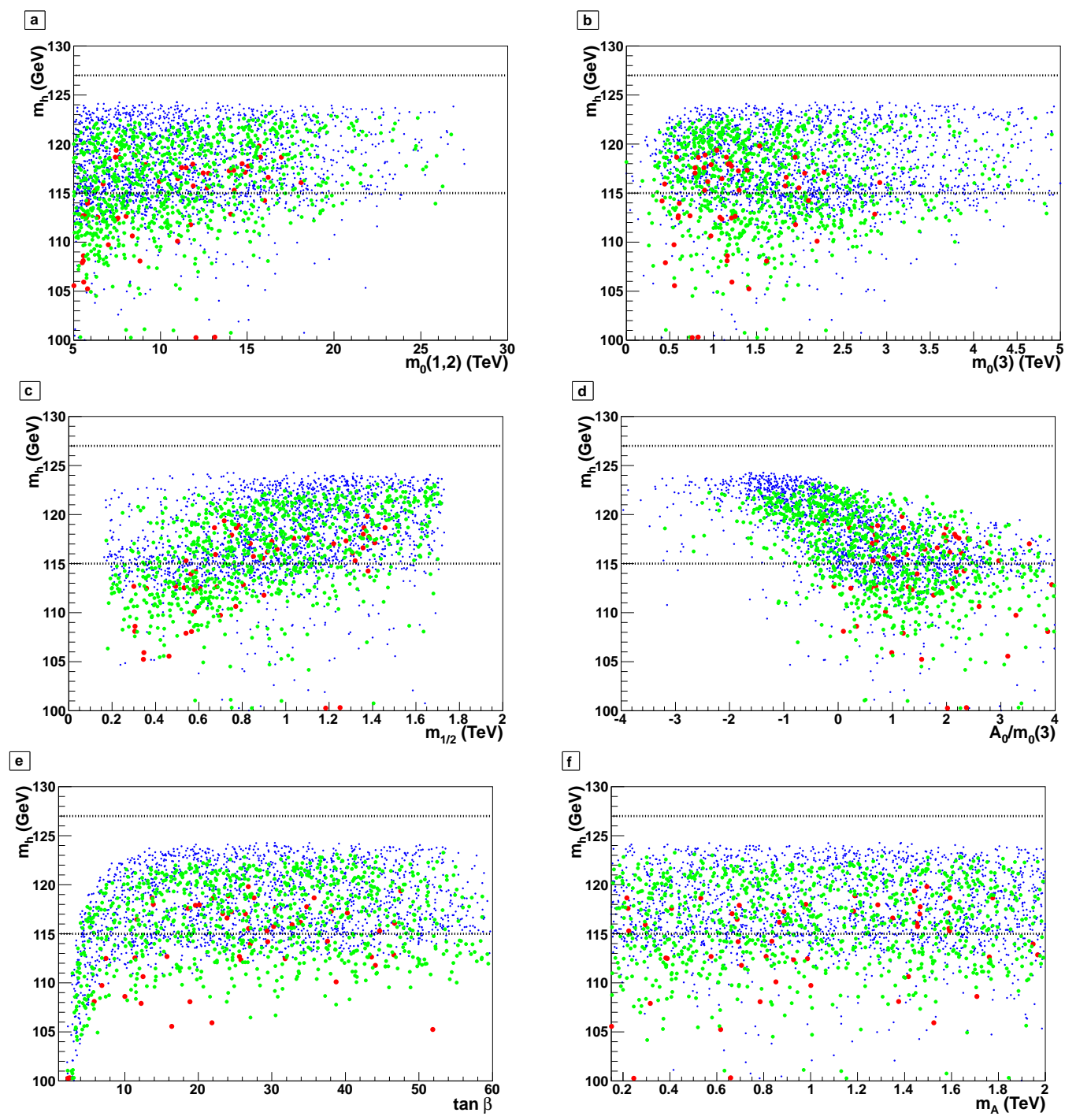

Figure 2: The value of $m_{h}$ versus various GUT scale SUSY parameters. Here, and in subsequent figures, the colour-coding is as in Fig. 1

to be compared with the measured value of $B F(b \rightarrow s \gamma)=(3.55 \pm 0.26) \times 10^{-4}$ from Ref. [44]. Indeed, as can be seen, large SUSY loop contributions cause the branching fraction to vary over a wide range: $(0-9) \times 10^{-4}$, so that many solutions would be rejected. Nonetheless, many other solutions do remain within the $\pm 3 \sigma$ band (which is shown), where the various loop contributions may cancel one against another to yield consistency with the measured value.

In addition, the well-known $(g-2)_{\mu}$ anomaly has been reported as a roughly $3 \sigma$ deviation from the SM value: $\Delta a_{\mu}=(28.7 \pm 8.0) \times 10^{-10}[45]$. In Natural SUSY, since the $\tilde{\mu}_{1,2}$ and $\tilde{\nu}_{\mu}$ masses are in the multi-TeV range, only a tiny non-standard contribution to the $(g-2)_{\mu}$ anomaly is expected, and alternative explanations for this anomaly would have to be sought. 


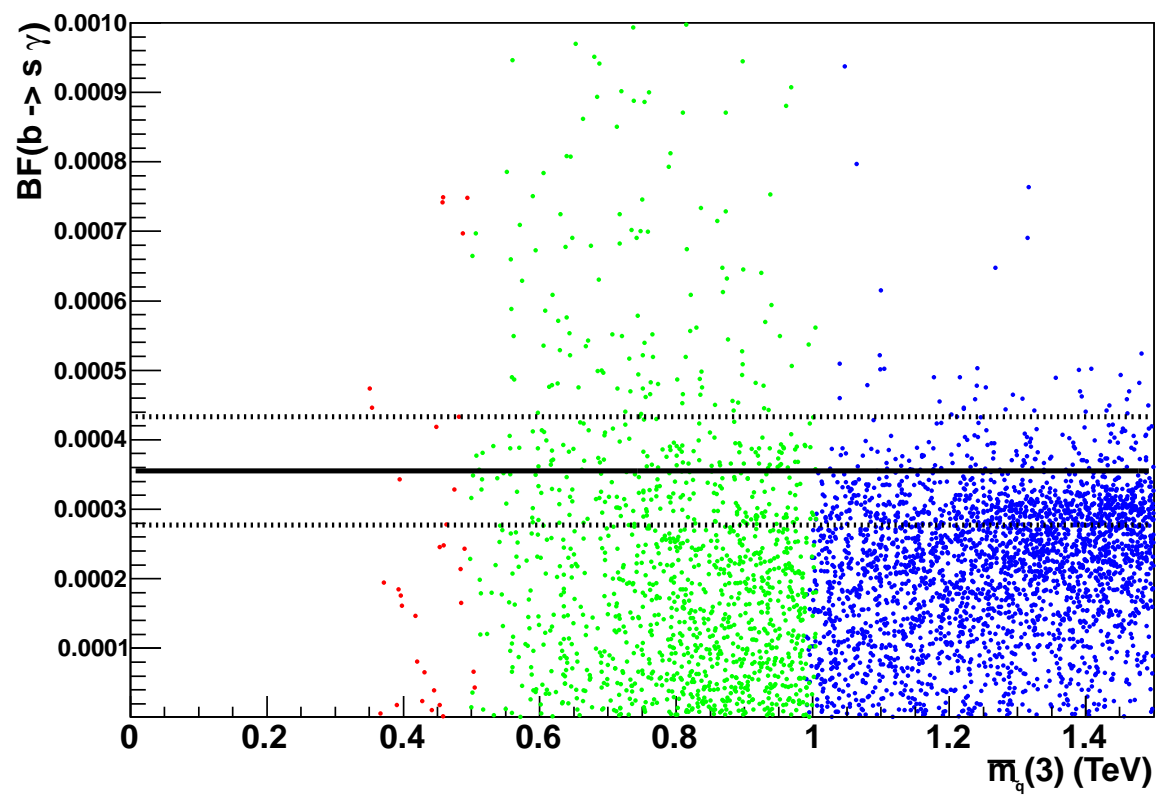

Figure 3: Predicted values of the branching fraction for $b \rightarrow s \gamma$ vs. $\bar{m}_{\tilde{q}}(3)$. We also show the experimentally determined central value $\pm 3 \sigma$ band for the $B F(b \rightarrow s \gamma)$.

\section{Benchmark points, slopes and planes}

In this section, we list some representative natural SUSY benchmark points, slopes and planes which could be used for LHC analyses. In Table 1, we show three such points, NS1 with $\bar{m}_{\tilde{q}}(3)=666 \mathrm{GeV}$, NS2 with $\bar{m}_{\tilde{q}}(3)=595 \mathrm{GeV}$ and NS3 with $\bar{m}_{\tilde{q}}(3)=1343.7 \mathrm{GeV}$. For all points, we fix $\mu=150 \mathrm{GeV}$, with large $m_{1 / 2}$ so that the higgsino-like chargino and the two lightest higgsino-like neutralinos have masses $\sim 150 \mathrm{GeV}$ and are the lightest sparticles. The light Higgs masses $m_{h} \sim 121 \mathrm{GeV}$ for the first two points, and so are low but as discussed above not incompatible with the recent hint for $m_{h} \sim 125 \mathrm{GeV}$. The third point NS3 allows $m_{h}=123.5 \mathrm{GeV}$ but at the expense of rather large $\bar{m}_{\tilde{q}}(3)$, and $m_{\tilde{t}_{2}}$ marginally beyond our naturalness requirement. For all these points the gluino mass is around $3 \mathrm{TeV}$ and first and second generation squarks are completely beyond the reach of the LHC.

For point NS1, the light top squark $\tilde{t}_{1}$ is next-lightest SUSY particle after the three higgsino-like states; it has mass $m_{\tilde{t}_{1}}=301.4 \mathrm{GeV}$ and may be accessible to LHC top squark searches. The $\tilde{b}_{1}$ and $\tilde{t}_{2}$ come in at 788 and $909 \mathrm{GeV}$, respectively. Both staus and the tau sneutrino are relatively light and might be accessible at a future $\mathrm{TeV}$-scale lepton-anti-lepton collider.

Point NS2 has a light bottom squark with $m_{\tilde{b}_{1}}=497.3 \mathrm{GeV}$ as next-lightest after the higgsinos. The $\tilde{t}_{1}$ is slightly heavier at $572 \mathrm{GeV}$. This point has heavier tau sleptons which would not be accessible to any planned lepton colliders. Point NS3 with rather heavy third generation squarks and sleptons would be very challenging to see at LHC although the 


\begin{tabular}{|c|c|c|c|}
\hline parameter & NS1 & NS2 & NS3 \\
\hline$m_{0}(1,2)$ & 13363.3 & 19542.2 & 7094.3 \\
\hline$m_{0}(3)$ & 761.1 & 2430.6 & 890.7 \\
\hline$m_{1 / 2}$ & 1380.2 & 1549.3 & 1202.6 \\
\hline$A_{0}$ & -167.0 & 873.2 & -2196.2 \\
\hline $\tan \beta$ & 22.9 & 22.1 & 19.4 \\
\hline$\mu$ & 150 & 150 & 150 \\
\hline$m_{A}$ & 1545.6 & 1652.7 & 410.1 \\
\hline$m_{\tilde{g}}$ & 3272.2 & 3696.8 & 2809.3 \\
\hline$m_{\tilde{u}_{L}}$ & 13591.1 & 19736.2 & 7432.9 \\
\hline$m_{\tilde{u}_{R}}$ & 13599.3 & 19762.6 & 7433.4 \\
\hline$m_{\tilde{e}_{R}}$ & 13366.1 & 19537.2 & 7086.9 \\
\hline$m_{\tilde{t}_{1}}$ & 301.4 & 572.0 & 812.5 \\
\hline$m_{\tilde{t}_{2}}$ & 909.2 & 715.4 & 1623.2 \\
\hline$m_{\tilde{b}_{1}}$ & 788.1 & 497.3 & 1595.5 \\
\hline$m_{\tilde{b}_{2}}$ & 1256.2 & 1723.8 & 1966.7 \\
\hline$m_{\tilde{\tau}_{1}}$ & 430.9 & 2084.7 & 652.2 \\
\hline$m_{\tilde{\tau}_{2}}$ & 532.9 & 2189.1 & 1065.5 \\
\hline$m_{\tilde{\nu}_{\tau}}$ & 402.3 & 2061.8 & 1052.1 \\
\hline$m_{\widetilde{W}_{2}}$ & 1180.2 & 1341.2 & 1013.9 \\
\hline$m_{\widetilde{W}_{1}}$ & 155.9 & 156.1 & 156.2 \\
\hline$m_{\widetilde{Z}_{4}}$ & 1181.3 & 1340.4 & 1020.0 \\
\hline$m_{\widetilde{Z}_{3}}$ & 615.3 & 698.8 & 532.6 \\
\hline$m_{\widetilde{Z}_{2}}$ & 156.3 & 156.2 & 157.0 \\
\hline$m_{\widetilde{Z}_{1}}$ & 148.4 & 149.2 & 147.4 \\
\hline$m_{h}$ & 121.3 & 121.1 & 123.5 \\
\hline$\Omega_{\widetilde{Z}_{1}}^{s t d} h^{2}$ & 0.007 & 0.006 & 0.007 \\
\hline$B F(b \rightarrow s \gamma)$ & $2.8 \times 10^{-4}$ & $3.6 \times 10^{-4}$ & $2.8 \times 10^{-4}$ \\
\hline$\sigma^{S I}\left(\widetilde{Z}_{1} p\right)(\mathrm{pb})$ & $5.5 \times 10^{-9}$ & $1.8 \times 10^{-9}$ & $9.8 \times 10^{-9}$ \\
\hline$\sigma^{S D}\left(\widetilde{Z}_{1} p\right)(\mathrm{pb})$ & $3.9 \times 10^{-5}$ & $2.9 \times 10^{-5}$ & $5.7 \times 10^{-5}$ \\
\hline$\left.\langle\sigma v\rangle\right|_{v \rightarrow 0}\left(\mathrm{~cm}^{3} / \mathrm{sec}\right)$ & $3.0 \times 10^{-25}$ & $3.1 \times 10^{-25}$ & $3.0 \times 10^{-25}$ \\
\hline
\end{tabular}

Table 1: Input parameters and masses in GeV units for three Natural SUSY benchmark points, with $\mu=150 \mathrm{GeV}$. Also shown are the values of several non-accelerator observables.

spectrum of light higgsinos should be accessible to a linear $e^{+} e^{-}$collider.

In Fig. 4, we convert benchmark point NS1 into a benchmark slope by retaining all parameters as in Table 1, except allowing $m_{0}(3)$ to vary. For $m_{0}(3)$ much below 700 $\mathrm{GeV}$, we generate spectra with tachyonic stops. Some gaps occur in the plot where no convergent RGE solution is found. These gaps can be filled in by increasing the number of iterations in Isasugra RGE running beyond the default value of 25 . In the Figure, we plot all four third generation squark masses versus $m_{0}(3)$, which gives a rising spectrum for 
most third generation squarks except the light top squark which reaches a minimal mass at $m_{0}(3) \simeq 840 \mathrm{GeV}$, where $m_{\tilde{t}_{1}}<m_{\widetilde{Z}_{1}}$ so that the $\tilde{t}_{1}$ is lightest MSSM particle. This point gives maximal mixing in the top squark sector, and a minimal value for $m_{\tilde{t}_{1}}$.

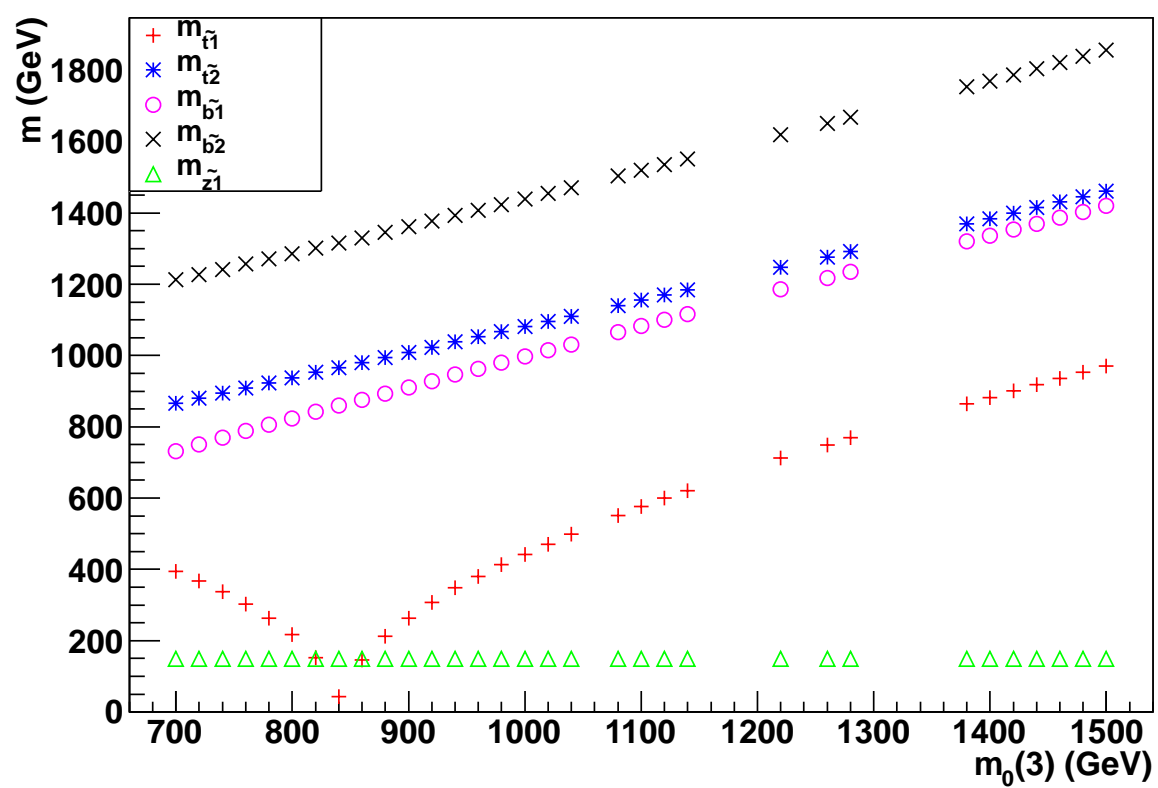

Figure 4: Plot of third generation squark masses together with the lightest neutralino mass versus variation in $m_{0}(3)$, with other parameters fixed as for the benchmark point NS1.

In Fig. 5, we convert benchmark point NS1 into a benchmark plane, where we plot contours of light top-squark mass $m_{\tilde{t}_{1}}$ as a function of $m_{0}(3) v s . \mu$ variation. The unshaded region gives rise to tachyonic squarks. Points with valid solutions are labeled as black dots; the gaps again require iterations beyond the default value of 25 . The color coding extrapolates the generated value of $m_{\tilde{t}_{1}}$, which again reaches a minimum of below $200 \mathrm{GeV}$ at $m_{0}(3) \sim 830 \mathrm{GeV}$.

\section{LHC signals for Natural SUSY}

We begin by noting that since $\mu \lesssim 200 \mathrm{GeV}$, we expect a spectrum of light, higgsino-like $\widetilde{W}_{1}, \widetilde{Z}_{1}$ and $\widetilde{Z}_{2}$ with mass $\sim \mu$ and small mass gaps $m_{\widetilde{W}_{1}}-m_{\widetilde{Z}_{1}} \sim m_{\widetilde{Z}_{2}}-m_{\widetilde{Z}_{1}} \sim 10-20$ GeV. Models with low $\mu$ parameter have been considered previously in Refs. 46] and 447. In Ref. [47, production cross sections for chargino pair production, chargino-neutralino production and neutralino pair production were presented. The -ino pair production cross sections tend to be in the 50-500 fb range. The decays $\widetilde{W}_{1} \rightarrow \widetilde{Z}_{1} f \bar{f}^{\prime}$ and $\widetilde{Z}_{2} \rightarrow \widetilde{Z}_{1} f \bar{f}$ (where $f$ collectively stands for light SM fermions) are dominated by $W^{*}$ and $Z^{*}$ exchange respectively. However, since the mass gaps $\widetilde{W}_{1}-\widetilde{Z}_{1}$ and $\widetilde{Z}_{2}-\widetilde{Z}_{1}$ are so small, there is only a small visible energy release in the decays, making the visible portion of the final state very soft and difficult to extract above SM backgrounds. In fact, models with low 


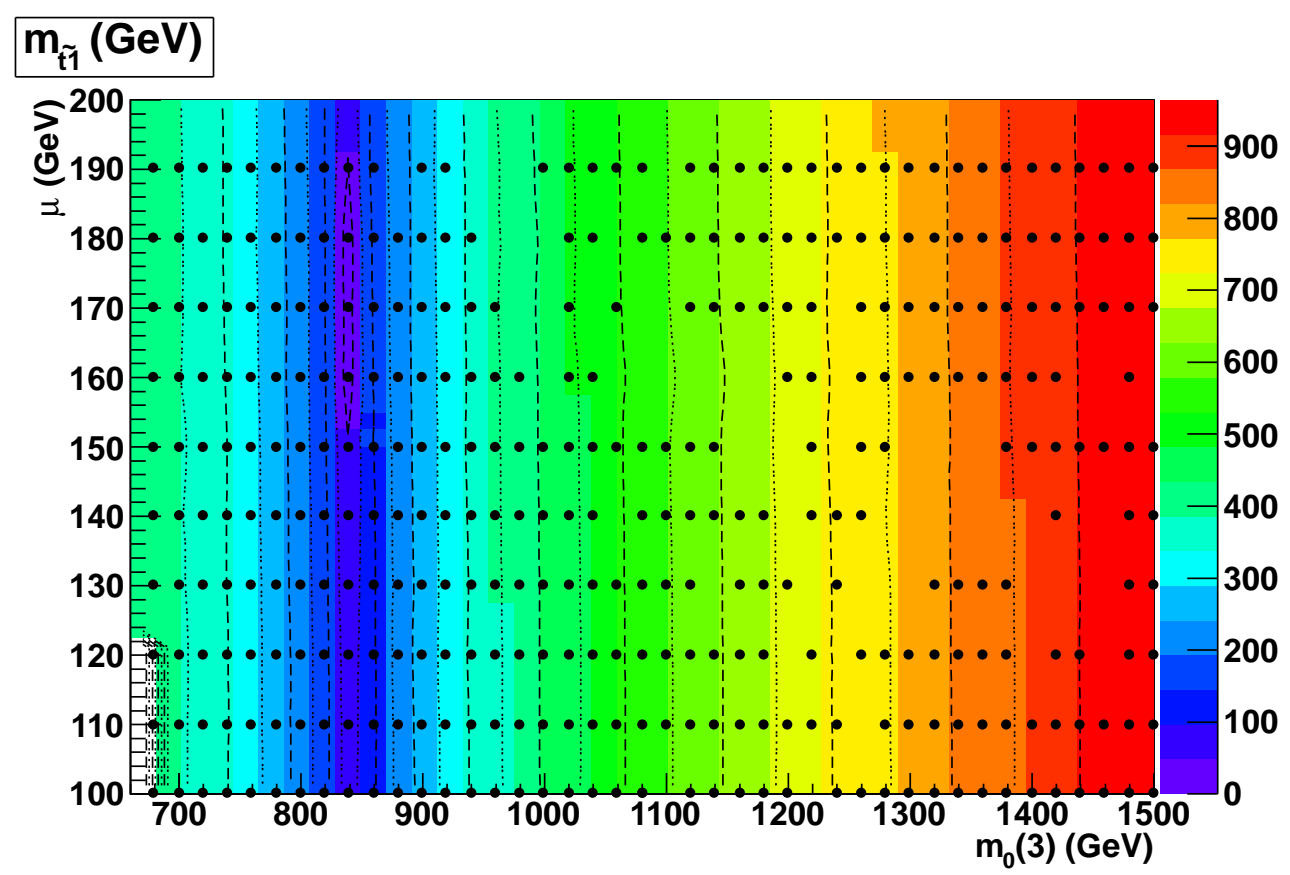

Figure 5: The top squark mass $m_{\tilde{t}_{1}}$ in the $\mu$ vs. $m_{0}(3)$ plane, with other parameters as for benchmark point NS1.

$\mu$ and concomitantly light higgsinos but other sparticles at the multi- $\mathrm{TeV}$ scale have been dubbed "hidden SUSY" in Ref. [47] because distinctive SUSY signals at LHC are extremely difficult to extract above background.

A key feature of Natural SUSY models is that they necessarily feature three and possibly four relatively light third generation squarks. While simplified models tend to focus on the signal from a single production mechanism, often assuming one dominant decay channel, generally speaking in natural SUSY we expect several third generation squarks to contribute to new physics signal rates. Moreover, these squarks will typically have decays to all three light higgsino-like states, and possibly also to other decay channels. While the lightest of these squarks will have the largest production cross sections, because of the larger mass gaps together with their more complex cascade decays, production of heavier third generation squarks may also yield observable signals.

In Fig. 6, we list the $p p \rightarrow \tilde{t}_{1} \tilde{\tilde{t}}_{1} X$ production cross section calculated in NLO QCD using Prospino 48]. We show results for LHC with $\sqrt{s}=7,8$ and $14 \mathrm{TeV}$ center-of-mass energy. ${ }^{6}$ The $\tilde{b}_{i} \tilde{b}_{i}$ (for $i=1,2$ ) and $\tilde{t}_{2} \overline{\tilde{t}}_{2}$ cross sections are essentially identical to those shown by making an appropriate mass substitution, since almost all the production cross section comes from light quark $q \bar{q}$ and $g g$ fusion in the initial state.

In Table 2, we show the various third generation squark pair production cross sections at LHC8 and branching fractions for benchmark points NS1, NS2 and NS3 from Table

\footnotetext{
${ }^{6}$ More precisely, because Prospino only allows a selection of Tevatron, LHC7 or LHC14 - but not LHC8 - we have obtained the cross section for LHC8 by scaling the corresponding Isajet cross section by the ratio of Prospino to Isajet cross sections for LHC7.
} 


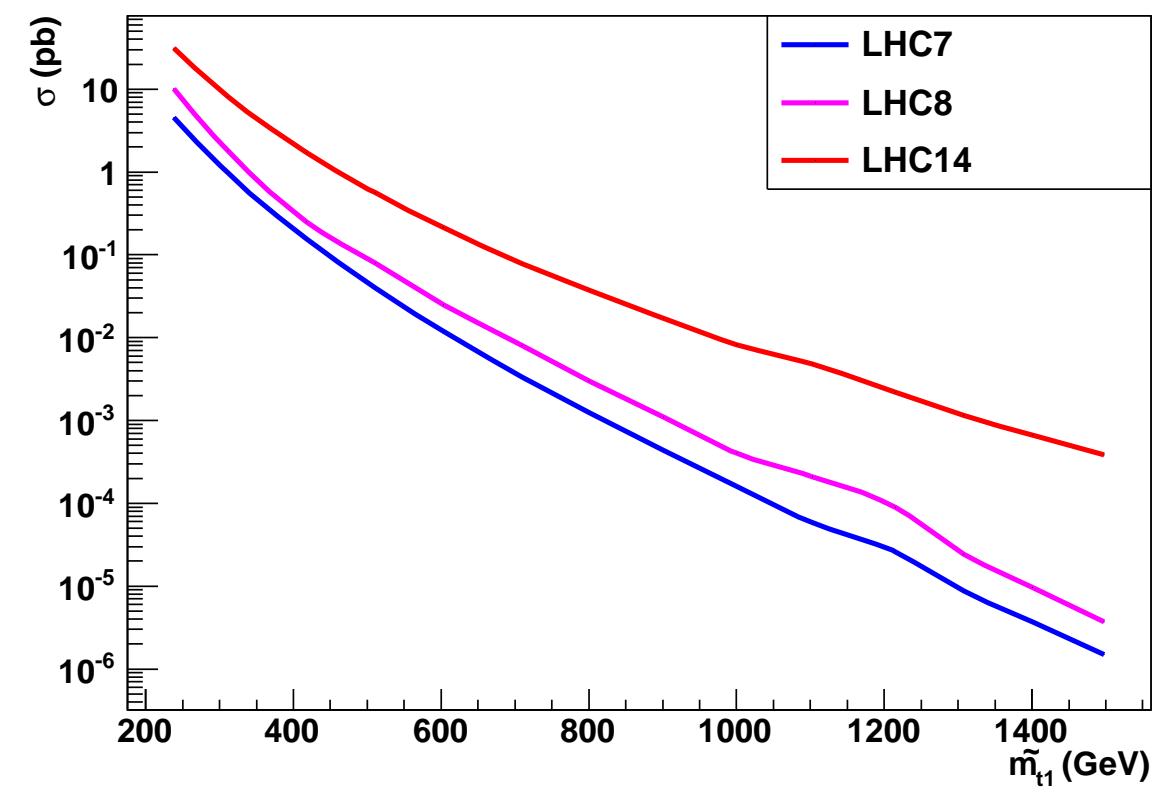

Figure 6: Top squark pair production cross sections at LHC7, LHC8 and LHC14 versus $m_{\tilde{t}_{1}}$. With the appropriate mass substitution these curves also represent the cross sections for pair production of $\tilde{t}_{2}, \tilde{b}_{1}$ or $\tilde{b}_{2}$ squarks.

1. Point NS1 is by far dominated by $\tilde{t}_{1} \overline{\tilde{t}}_{1}$ production at LHC 8 with a cross section of $\sim 2$ pb. The $\tilde{t}_{1}$ then decays to $b \widetilde{W}_{1}$ at $\sim 100 \%$ branching fraction. This model would be well-described by a simplified model analysis, where the final state $\widetilde{W}_{1}$ is essentially regarded as missing- $E_{T}$ due to its soft decay products. Thus, the signature would be a pair of acollinear $b$-jets together with $E_{T}^{\text {miss }}$ and no other transverse activity except from QCD radiation. The dominant SM physics background would be from $Z b \bar{b}$ production, with $Z \rightarrow \nu \bar{\nu}$. At LHC8, there is also a $4 \mathrm{fb}$ cross section from $\tilde{b}_{1} \overline{\tilde{b}}_{1}$ production followed by $\tilde{b}_{1} \rightarrow W \tilde{t}_{1}$, giving rise to $b \bar{b} W^{+} W^{-}+E_{T}^{\text {miss }}$ events, albeit at low rates. These would be subject to a daunting background from $t \bar{t}$ production. We mention that at LHC14, $\tilde{t}_{2}$-pair production which has a cross section of $\sim 20 \mathrm{fb}$ could lead to a handful of spectacular $\tilde{t}_{2} \overline{\tilde{t}}_{2} \rightarrow Z Z \tilde{t}_{1} \overline{\tilde{t}}_{1} \rightarrow Z Z b \bar{b}+E_{T}^{\text {miss }}$ events where the $Z$ s might be identified via their leptonic decays.

For the point NS2, $p p \rightarrow \tilde{b}_{1} \overline{\tilde{b}}_{1}$ production is dominant at $\sigma \sim 80 \mathrm{fb}$, although $\tilde{t}_{1} \overline{\tilde{t}}_{1}$ is also produced at $\sim 30 \mathrm{fb}$. In this case, the $\tilde{b}_{1}$ decays dominantly via $\tilde{b}_{1} \rightarrow t \widetilde{W}_{1}$ giving rise to a $t \bar{t}+E_{T}^{\text {miss }}$ signature at LHC. The decays $\tilde{b}_{1} \rightarrow b \widetilde{Z}_{1}$ and $\tilde{b}_{1} \rightarrow b \widetilde{Z}_{2}$ also occur at $\sim 10 \%$ level. The $\tilde{t}_{1}$ decay modes are spread somewhat evenly between $b \widetilde{W}_{1}, t \widetilde{Z}_{1}$ and $t \widetilde{Z}_{2}$ final states. Again, the small mass gap between the $\widetilde{W}_{1} / \widetilde{Z}_{2}$ and the LSP implies that the chargino and the neutralino daughters are essentially invisible. By combining all modes, the most lucrative signature channels consist of $b \bar{b}+E_{T}^{\text {miss }}$ and $t \bar{t}+E_{T}^{\text {miss }}$ events. The heavier $\tilde{t}_{2}$ decay modes are spread among many more possibilities, including decays to $W$ and $h$ bosons in the final state; a handful of novel events may be obtained at LHC8, but 


\begin{tabular}{|c|c|c|c|}
\hline parameter & NS1 & NS2 & NS3 \\
\hline$\sigma\left(\tilde{t}_{1} \tilde{t}_{1}\right)$ & $2000 \mathrm{fb}$ & $30 \mathrm{fb}$ & $2 \mathrm{fb}$ \\
\hline$B F\left(\tilde{t}_{1} \rightarrow b \widetilde{W}_{1}\right)$ & 1.0 & 0.25 & 0.62 \\
\hline$B F\left(\tilde{t}_{1} \rightarrow t \widetilde{Z}_{1}\right)$ & - & 0.42 & 0.08 \\
\hline$B F\left(\tilde{t}_{1} \rightarrow t \widetilde{Z}_{2}\right)$ & - & 0.33 & 0.30 \\
\hline$\sigma\left(\tilde{b}_{1} \tilde{b}_{1}\right)$ & $4 \mathrm{fb}$ & $80 \mathrm{fb}$ & $0.00013 \mathrm{fb}$ \\
\hline$B F\left(\tilde{b}_{1} \rightarrow b \widetilde{Z}_{1}\right)$ & 0.01 & 0.10 & 0.01 \\
\hline$B F\left(\tilde{b}_{1} \rightarrow b \widetilde{Z}_{2}\right)$ & 0.01 & 0.09 & 0.01 \\
\hline$B F\left(\tilde{b}_{1} \rightarrow t \widetilde{W}_{1}\right)$ & 0.09 & 0.81 & 0.04 \\
\hline$B F\left(\tilde{b}_{1} \rightarrow W \tilde{t}_{1}\right)$ & 0.89 & - & 0.94 \\
\hline$\sigma\left(\tilde{t}_{2} \tilde{t}_{2}\right) \quad \sim$ & $1 \mathrm{fb}$ & $6 \mathrm{fb}$ & $0.00011 \mathrm{fb}$ \\
\hline$B F\left(\tilde{t}_{2} \rightarrow b \widetilde{W}_{1}\right)$ & 0.09 & 0.29 & 0.05 \\
\hline$B F\left(\tilde{t}_{2} \rightarrow Z \tilde{t}_{1}\right)$ & 0.70 & 0.01 & 0.39 \\
\hline$B F\left(\tilde{t}_{2} \rightarrow h \tilde{t}_{1}\right)$ & 0.01 & 0.23 & 0.25 \\
\hline$B F\left(\tilde{t}_{2} \rightarrow W \tilde{b}_{1}\right)$ & 0.03 & 0.16 & 0.26 \\
\hline$B F\left(\tilde{t}_{2} \rightarrow t \widetilde{Z}_{1}\right)$ & 0.09 & 0.13 & 0.03 \\
\hline$B F\left(\tilde{t}_{2} \rightarrow t \widetilde{Z}_{2}\right)$ & 0.08 & 0.16 & 0.02 \\
\hline$\sigma\left(\tilde{b}_{2} \tilde{b}_{2}\right)$ & $0.05 \mathrm{fb}$ & $0.0001 \mathrm{fb}$ & $0.00004 \mathrm{fb}$ \\
\hline$B F\left(\tilde{b}_{2} \rightarrow b \widetilde{Z}_{1}\right)$ & 0.22 & 0.23 & 0.01 \\
\hline$B F\left(\tilde{b}_{2} \rightarrow b \widetilde{Z}_{2}\right)$ & 0.22 & 0.22 & 0.01 \\
\hline$B F\left(\tilde{b}_{2} \rightarrow b \widetilde{Z}_{3}\right)$ & 0.07 & 0.08 & - \\
\hline$B F\left(\tilde{b}_{2} \rightarrow t \widetilde{W}_{1}\right)$ & 0.42 & 0.44 & 0.02 \\
\hline$B F\left(\tilde{b}_{2} \rightarrow W \tilde{t}_{1}\right)$ & 0.03 & 0.01 & - \\
\hline$B F\left(\tilde{b}_{2} \rightarrow h \tilde{b}_{1}\right)$ & 0.03 & 0.02 & - \\
\hline$B F\left(\underset{\sim}{\tilde{b}_{2}} \rightarrow H \tilde{b}_{1}\right)$ & - & - & 0.23 \\
\hline$B F\left(\tilde{b}_{2} \rightarrow A \tilde{b}_{1}\right)$ & - & - & 0.23 \\
\hline$B F\left(\tilde{b}_{2} \rightarrow H^{-} \tilde{t}_{1}\right)$ & - & - & 0.41 \\
\hline$B F\left(\tilde{b}_{2} \rightarrow H^{-} \tilde{t}_{2}\right)$ & - & - & 0.08 \\
\hline
\end{tabular}

Table 2: Production cross sections at LHC8 and branching fractions for third generation squark production for the Natural SUSY benchmark points from Table 1.

more likely at LHC14. The $\tilde{b}_{2}$ state appears likely undetectable even at LHC14.

For the benchmark point NS3, the detection of third generation squarks at LHC8 appears to be very difficult on account of the very low cross sections. Even at LHC14, the cross section for $\tilde{t}_{1} \tilde{t}_{1}$ production is just $50 \mathrm{fb}$, and the fact that the chargino and neutralino daughters are (nearly) invisible will make identification of the acollinear $t \bar{t}$ and $b \bar{b}$ events from this quite challenging. Production of $\tilde{t}_{2} \overline{\tilde{t}}_{2}$ and $\tilde{b}_{1} \overline{\tilde{b}}_{1}$ at LHC14 occurs at a fraction of a fb level, though the interesting topologies that include $Z$ and $h$ production from $\tilde{t}_{2}$ cacsade decays may be accessible at super-LHC luminosities.

We would also like to assess the prospects for discovering the gluino of the natural SUSY framework at the LHC. A plot of $m_{\tilde{g}} v s . \bar{m}_{\tilde{q}}(3)$ is shown in Fig. 0 for scan points 
fulfilling the $B F(b \rightarrow s \gamma)$ constraint and also $m_{h}>115 \mathrm{GeV}$. From the plot, we see that while models with $m_{\tilde{g}} \lesssim 1 \mathrm{TeV}$ can be readily obtained for $\bar{m}_{\tilde{q}}(3) \sim 1-1.5 \mathrm{TeV}$, a tighter restriction of $\bar{m}_{\tilde{q}}(3)<0.5(1 \mathrm{TeV})$ typically limits $m_{\tilde{g}} \gtrsim 2 \mathrm{TeV}(1 \mathrm{TeV})$. In the case of models with multi-TeV squarks, the LHC8 reach (which should be close to LHC7 reach [49]) for $\sim 20 \mathrm{fb}^{-1} \mathrm{fb}$ of integrated luminosity extends to $m_{\tilde{g}} \sim 1 \mathrm{TeV}$. The LHC14 reach[50] for $100 \mathrm{fb}^{-1}$ extends to $\sim m_{\tilde{g}} \sim 1.8 \mathrm{TeV}$. These studies have been done within the mSUGRA model, and for LHC14 without $b$-jet tagging which should enhance the SUSY signal in Natural SUSY models. The increased reach in the gluino mass is projected to be up to $20 \%$, depending on the details of the particle spectrum [51]. We conclude that while some models with large $\bar{m}_{\tilde{q}}(3)>1 \mathrm{TeV}$ may be accessible to LHC gluino searches, ${ }^{7}$ there remain many models (expecially for low $\bar{m}_{\tilde{q}}(3)<1 \mathrm{TeV}$ ) where gluino pair production will be beyond even the LHC14 reach.

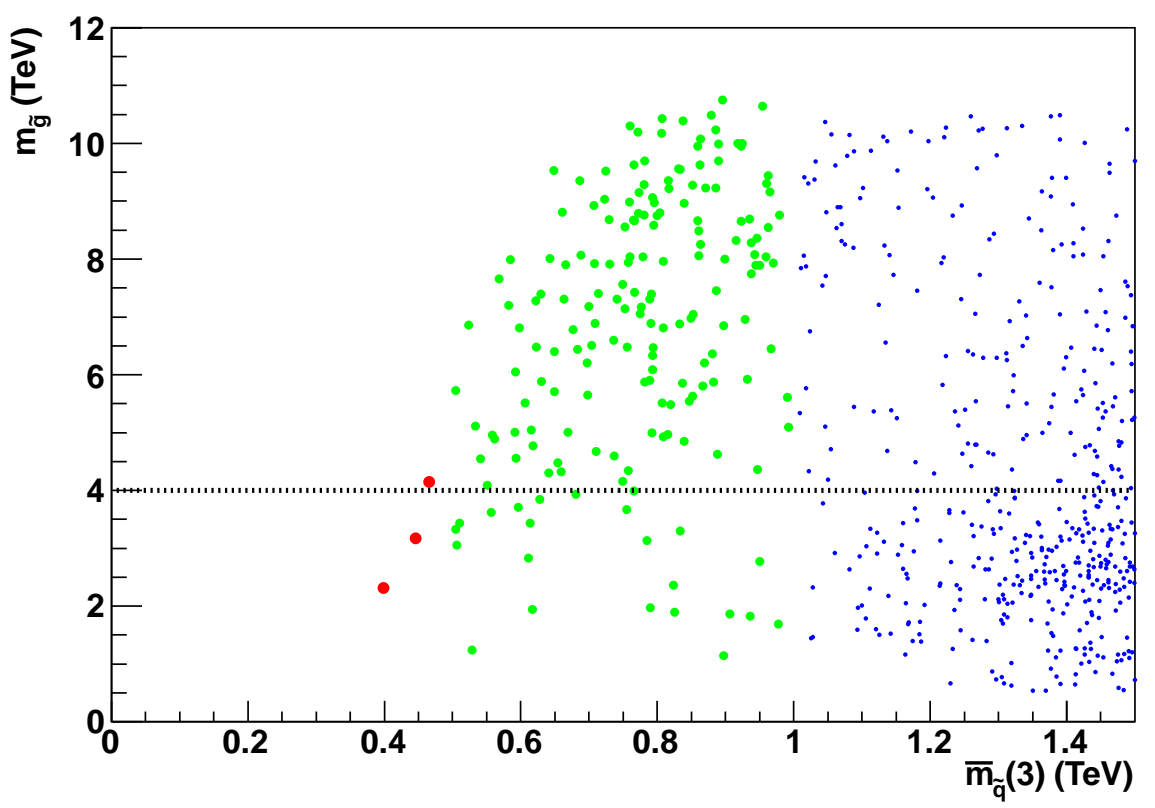

Figure 7: Value of $m_{\tilde{g}} v s . \bar{m}_{\tilde{q}}(3)$ from Natural SUSY models which obey $B F(b \rightarrow s \gamma)$ at $3 \sigma$ and $m_{h}>115 \mathrm{GeV}$.

Lastly, motivated by the bound on $m_{A}$ presented in Sec. 1.3, we plot $m_{A} v s \bar{m}_{\tilde{q}}(3)$ in Fig. 8 for natural SUSY points with $m_{\tilde{g}}<4 \mathrm{TeV}, m_{h}>115 \mathrm{GeV}$ and which satisfy the $B(b \rightarrow s \gamma)$ constraint. The color coding is as in Fig. Q7. We see that $m_{A} \gtrsim 500 \mathrm{GeV}$ for low $\bar{m}_{\tilde{q}}(3)$ values, while $m_{A}$ can be as low as a few hundred $\mathrm{GeV}$ for very large $\bar{m}_{\tilde{q}}(3)$.

\footnotetext{
${ }^{7}$ In this context, we note that the ATLAS LHC7 limits [52] from gluino-mediated stop-pair searches do not directly apply because these rely on the same-sign dilepton signal where the lepton may arise from either the top quark daughter of the gluino or from the chargino daughter of the top squark. In our case, we expect leptons from the latter source to be very soft.
} 


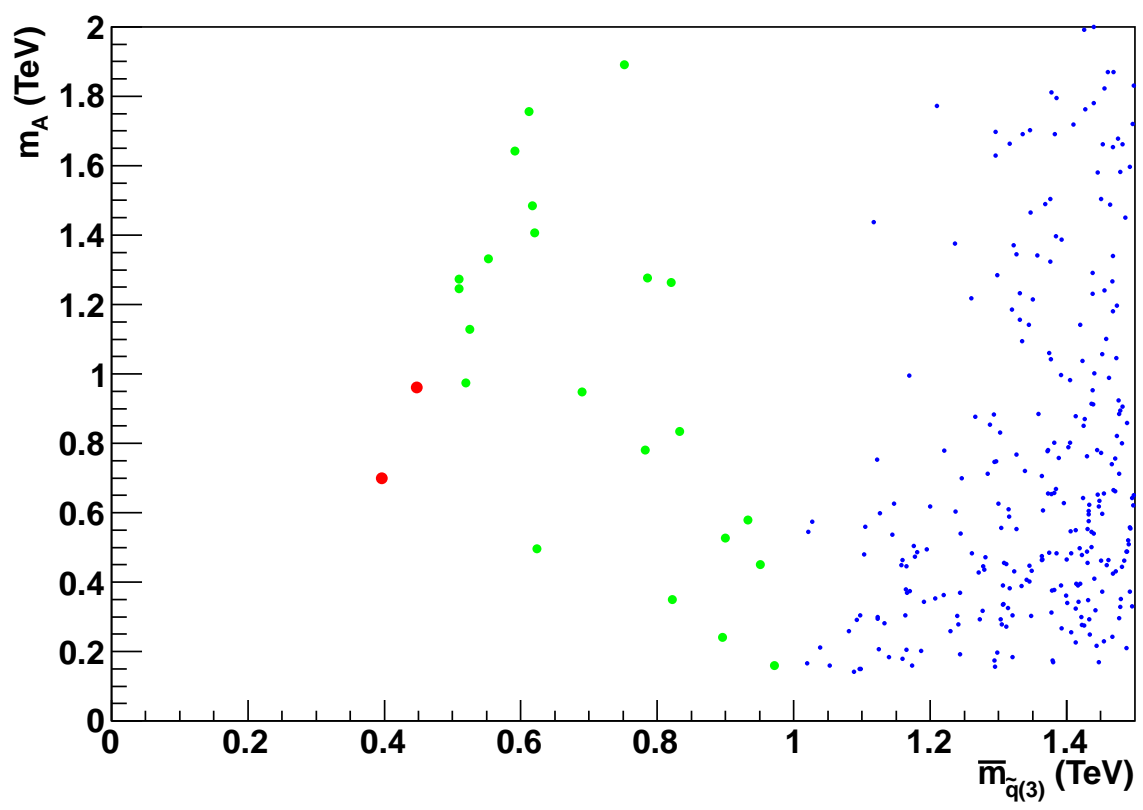

Figure 8: Value of $m_{A} v s . \bar{m}_{\tilde{q}}(3)$ in Natural SUSY models with $m_{\tilde{g}}<4 \mathrm{TeV}, m_{h}>115 \mathrm{GeV}$ and which satisfy $B F(b \rightarrow s \gamma)$ at $3 \sigma$. The color coding is as in Fig. 7 .

\section{Natural SUSY at a linear $e^{+} e^{-}$collider}

While Natural SUSY may possibly be difficult to discover at the LHC, it leads to a potential bonanza of signals for a linear $e^{+} e^{-}$collider (LC) operating in the $0.3-1 \mathrm{TeV}$ range. A LC would potentially be a higgsino factory because, as we have emphasized, the $\mu$ parameter is necessarily small in this scenario. Indeed, pair production of higgsino-like chargino and also neutralino states $\widetilde{W}_{1}^{ \pm}, \widetilde{Z}_{1}$ and $\widetilde{Z}_{2}$ with sizeable cross sections is inevitable at a $0.25-0.5 \mathrm{TeV}$ machine. Example cross section plots have been shown in Ref. [47] and so will not be repeated here. Thus, a natural target for a LC would be the pair production reactions $e^{+} e^{-} \rightarrow \widetilde{W}_{1}^{+} \widetilde{W}_{1}^{-}, \widetilde{Z}_{1} \widetilde{Z}_{2}$ and $\widetilde{Z}_{2} \widetilde{Z}_{2}$. While the small $\widetilde{W}_{1}-\widetilde{Z}_{1}$ and $\widetilde{Z}_{2}-\widetilde{Z}_{1}$ mass gaps are a formidable challenge at LHC (and may also be problematic at a LC), it has been shown [53] that with specialized cuts, it should be possible to extract a signal above SM background at a LC. The visible energy from these reactions would be low just above threshold, but as $\sqrt{s}$ increases, the decay products from $\widetilde{W}_{1}$ and $\widetilde{Z}_{2}$ would be boosted to higher energies. In addition, the beam polarization would be a strong tool not only for distinguishing the signal from $W^{+} W^{-}$backgrounds, but also for distinguishing between wino-like versus higgsino-like charginos 47.

In the case of the NS1 benchmark, after the light higgsinos are well studied and the CM energy $\sqrt{s}$ is increased, the next target threshold would be $\tilde{t}_{1} \overline{\tilde{t}}_{1}$ production at $\sqrt{s} \sim$ $2 m_{\tilde{t}_{1}} \sim 610 \mathrm{GeV}$. This would be followed by the tau-snuetrino pair production threshold at $\sqrt{s} \sim 810 \mathrm{GeV}$, with $\tilde{\nu}_{\tau} \rightarrow \widetilde{W}_{1}^{+} \tilde{\tau}_{1}^{-}$decay. At a little higher energy, $\tau_{1}^{+} \tilde{\tau}_{1}^{-}$pair production would turn on, followed mainly by $\tilde{\tau}_{1} \rightarrow \widetilde{Z}_{1} \tau$ and $\widetilde{Z}_{2} \tau$ decay. For the heavier spectra shown 
in NS2 and NS3, the light higgsino pair production reactions would still be available, but $\mathrm{CM}$ energies of over $1 \mathrm{TeV}$ would be required to pick up any squark pair production reactions. As emphasized above, the accessibility of higgsino-like states is a generic feature of Natural SUSY models.

\section{Natural SUSY and direct/indirect WIMP searches}

As noted in Sec. 1, the higgsino-like neutralinos with masses $\sim 100-200 \mathrm{GeV}$ expected in NS models annihilate very rapidly in the early universe and so yield a thermal relic underabundance of CDM. However, the neutralino relic abundance can be boosted to match its observed value in models where

- a PQ solution to the strong CP problem is invoked, and thermally-produced but latedecaying axino (and/or saxion) decays augment the SUSY particle production 27, 28], or

- there exist late-decaying $\mathrm{TeV}$ scale moduli fields with large branching fractions to SUSY particles that subsequently decay to the neutralino[54].

We stress that it in the first case it is not necessary that neutralinos saturate the observed relic density. Indeed it is easy to select PQ parameters where the converse is true: $\Omega_{\widetilde{Z}_{1}} h^{2}$ stays low while the bulk of dark matter is comprised of axions, or even where both axion and neutralino abundances are comparable. In this case, the direct and indirect neutralino reach estimates presented below (these have been obtained assuming that neutralinos saturate the CDM density) would have to be increased by a factor of $0.1123 / \Omega_{\widetilde{Z}_{1}} h^{2}$. It is difficult, but not impossible 28, to lower the neutralino abundance below its standard thermally produced prediction. Thus, we expect the above reach scale factor to be typically between 1 and 16, since $\Omega_{\widetilde{Z}_{1}}^{\text {thermal }} h^{2} \sim 0.007$ for a higgsino-like relic neutralino with a mass $\sim 150 \mathrm{GeV}$.

In Fig. 9, we show the spin-independent $\widetilde{Z}_{1} p$ scattering cross section in $p b$ as obtained from IsaReS 55. Here, and in the remainder of this section we assume that $\widetilde{Z}_{1}$ saturates the DM density. We plot points versus $\bar{m}_{\tilde{q}}(3)$, since $m_{\widetilde{Z}_{1}}$ is fixed typically $\sim 150 \mathrm{GeV}$ due to our choice of $\mu=150 \mathrm{GeV}$. We actually find that the bulk of points inhabit the $\gtrsim 10^{-8}$ pb range. Comparing to the bound from Xe-100[56], we find that a large fraction of these points are excluded if the higgsino-like WIMP is essentially all the dark matter. Moreover, with this same assumption, a large fraction of surviving points lie within the projected reach of Xe-100/2012 run, and certainly within the reach of Xe-1ton.

In Fig. 10, we plot the spin-dependent $\sigma^{S D}\left(\widetilde{Z}_{1} p\right)$ scattering cross section, this time in comparison to current and future IceCube reach [57], and future COUPP reach [58]. While the current IceCube reach excludes a significant portion of points (under the asumption of neutralino dominance), the future IceCube and especially COUPP reaches will access most of the remaining parameter space.

Fig. 11 shows the thermally averaged neutralino annihilation cross section times relative velocity, evaluated as $v \rightarrow 0$. This quantity enters linearly into indirect searches for 


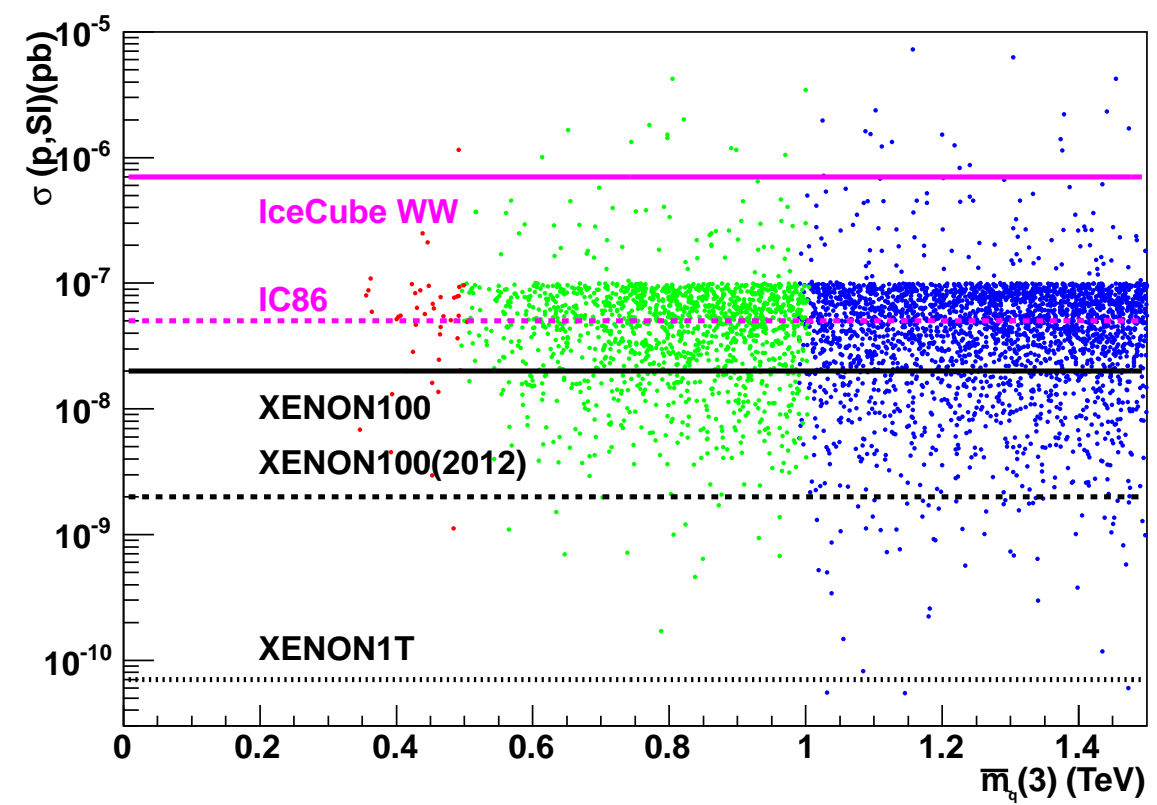

Figure 9: Spin independent $p \widetilde{Z}_{1}$ scattering cross section versus $m_{0}(3)$ for NS models with $\mu=150$ $\mathrm{GeV}$. Also shown are the current 90\% CL bounds together with projections from the XENON100 (2012 sensitivity) and IceCube (180 day sensitivity) experiments for a $150 \mathrm{GeV}$ WIMP (assuming higgsino-like WIMPs saturate the measured dark matter abundance).

neutralino annihilation in the cosmos into $\gamma \mathrm{s}$ or $e^{+}, \bar{p}$ or $\bar{D}$ searches. For our case, the bulk of points inhabit the range $\left.\langle\sigma v\rangle\right|_{v \rightarrow 0} \gtrsim 10^{-25} \mathrm{~cm}^{3} / \mathrm{sec}$. The horizontal solid line shows the upper limit on the annihilation cross section times velocity for very non-relativisitic dark matter in dwarf spheroidal satellite galaxies of the Milky Way annihilating to $W$ boson pairs obtained by the Fermi collaboration 59], assuming a $~ 150 \mathrm{GeV}$ WIMP. Models with a larger annihilation cross section would have led to a flux of gamma rays not detected by the experiment, assuming a Navarro-Frenk-White profile for each dwarf galaxy in the analysis. We see that the Fermi bound excludes the bulk of points for our choice of DM mass, again assuming higgsinos saturate the DM density. Moreover, this bound changes rather slowly with the DM mass, being just a factor of 2 weaker for a WIMP mass of $300 \mathrm{GeV}$. Further searches and improvements by the Fermi-LAT Collaboration and/or the impending AMS results should provide an incisive probe of the NS framework.

\section{Summary and conclusions}

The Natural SUSY model is defined by distinctive spectra characterized by a low $|\mu| \sim$ $m_{h} \lesssim 150-200 \mathrm{GeV}$, with a rather light spectrum of third generation squarks $m_{\tilde{t}_{1}}, m_{\tilde{t}_{2}}, m_{\tilde{b}_{1}} \lesssim$ $0.5-1.5 \mathrm{TeV}$ to stabilize the electroweak scale. In addition, $m_{\tilde{g}} \lesssim 4 \mathrm{TeV}$ so that loop corrections to third generation squark masses are smaller than the squark mass. First/second generation sfermions, on the other hand, could be at the tens of $\mathrm{TeV}$ scale, thus suppress- 


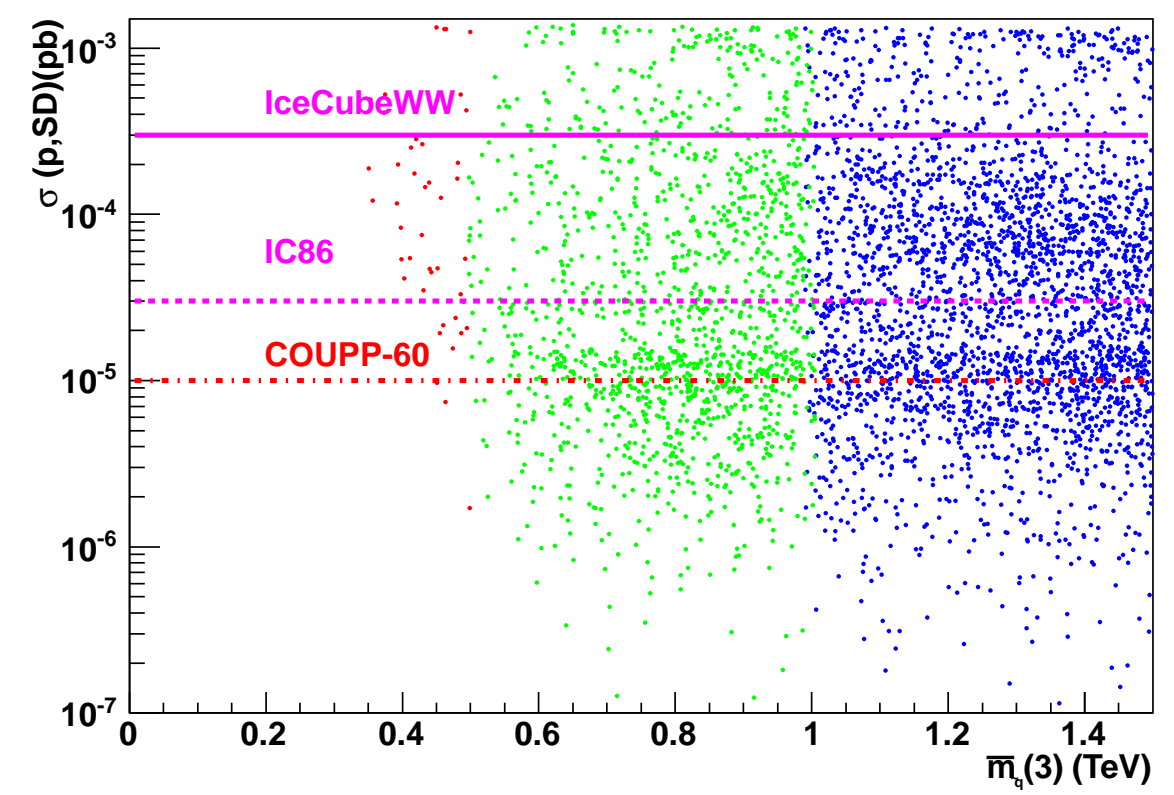

Figure 10: Spin dependent $p \widetilde{Z}_{1}$ scattering cross section versus $m_{0}(3)$ for NS models with $\mu=150$ $\mathrm{GeV}$. Also shown are current limits and future projections from IceCube and COUPP experiments (assuming higgsino-like WIMPs saturate the measured dark matter abundance).

ing unwanted flavor-violating and $C P$-violating processes. Motivated by gauge coupling unification, we expect the MSSM, or MSSM plus gauge singlets, to be the effective field theory between $M_{\text {weak }}$ and $M_{\mathrm{GUT}}$. In this case, the Natural SUSY mass spectra should arise from underlying fundamental parameters that have their origin in GUT scale physics. In this paper, we determine the values of GUT scale parameters which lead to models of natural SUSY. We find that, at the GUT scale,

- third generation mass parameters, $m_{0}(3) \sim 0.5-4 \mathrm{TeV}$,

- first/second generation mass parameters, $m_{0}(1,2) \sim 5-25 \mathrm{TeV}$,

- unified gaugino mass parameters, $m_{1 / 2} \sim 0.3-1.7 \mathrm{TeV}$, and

- the trilinear (third generation) scalar coupling, $A_{0} / m_{0}(3) \gtrsim-2$

yield models with a natural SUSY spectrum. The range of $\tan \beta$ and $m_{A}$ are relatively unrestricted. Note that there is an upper bound on $m_{0}(1,2)$ : values much larger than about $25 \mathrm{TeV}$ push third generation squarks into the tachyonic range via 2-loop RGE effects. We also find that values of $m_{h} \sim 125 \mathrm{GeV}$ are very difficult to reconcile with a spectrum with very light third generation scalars $\left(\bar{m}_{\tilde{q}}(3)<0.5 \mathrm{TeV}\right)$, but values of $m_{h}$ up to $124 \mathrm{GeV}$ can be realized if we allow $\bar{m}_{\tilde{q}}(3)$ up to $1-1.5 \mathrm{TeV}$ instead. Since some third generation squarks and charginos are rather light in natural SUSY, the constraint from $B F(b \rightarrow s \gamma)$ is rather strong, but models can be found which are consistent with the measured branching fraction. We provide some representative benchmark points for low and high values of $m_{h}$. 


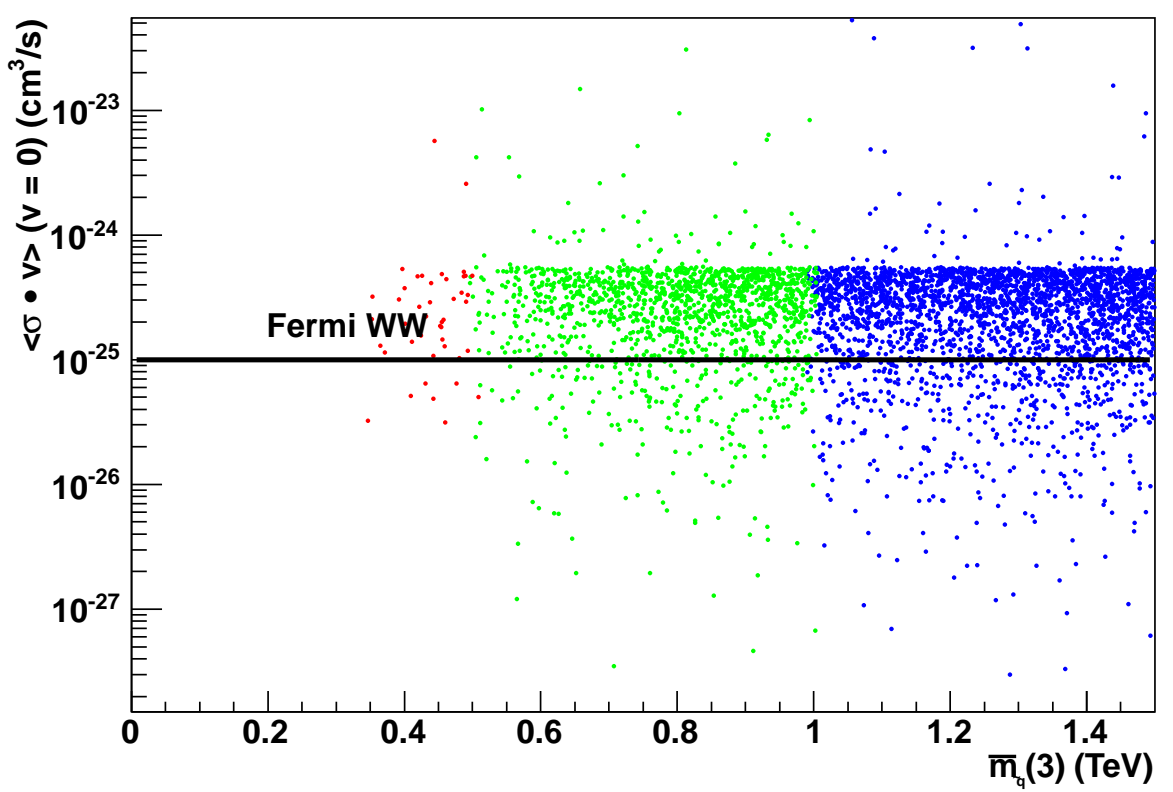

Figure 11: Thermally averaged neutralino annihilation cross section times relative velocity in limit as $v \rightarrow 0$ versus $m_{0}(3)$ for NS models with $\mu=150 \mathrm{GeV}$ together with the bound from the Fermi satellite on the cross section times velocity for WIMP annihilation to $W$-pairs (assuming higgsino-like WIMPs saturate the measured dark matter abundance).

At the LHC, the higgsino-like light charginos and neutralinos have only small energy release in their decays, and so will be difficult to observe, as noted for the related "hidden SUSY" scenario 47. However, in the case of natural SUSY, all four third generation squarks may be produced at observable rates. Sometimes, the lightest of these may have just one decay mode accessible (e.g. case NS1 in this paper), and thus may be described by an analysis using simplified models (this is essentially impossible if $\tilde{b}_{1}$ is the lightest squark). However, other cases arise where several different cascade decay possibilities are open. The heavier third generation squarks decay via numerous modes, and could lead to novel signatures involving $h$ or $Z$ from their cascade decays. Gluinos are favored to be rather heavy and frequently beyond LHC reach, although cases where $m_{\tilde{g}} \lesssim 1.5 \mathrm{TeV}$ do occur especially for $\bar{m}_{\tilde{q}}(3) \sim 1-1.5 \mathrm{TeV}$.

At a linear $e^{+} e^{-}$collider, we expect pair production of the higgsino-like light charginos and neutralinos to offer a lucrative discovery program of physics though specialized search strategies will be needed to pull out the rather soft signal events. In addition, it is possible that several third generation squarks and sleptons may be accessible to a LC with $\sqrt{s}$ extending up to $\sim 1 \mathrm{TeV}$ or beyond. Although these may decay to the light chargino as well as two lighter neutralinos, it will be challenging to sort out the various signals from the electroweak-ino cascades with very small secondary mass gaps. To our knowledge there are no dedicated studies for event topologies with this novel spectrum.

In Natural SUSY models, the higgsino-like neutralino $\widetilde{Z}_{1}$ is lightest MSSM particle, 
and standard relic density calculations predict an underabundance of higgsino-like WIMPS by a factor typically 15 . Such an under-abundance can be easily boosted to higher values if 1. there are late decaying moduli fields with large branching fractions to SUSY particles, or 2 . if the $\mathrm{PQ}$ solution to the strong $C P$ problem is invoked, whereupon thermal production of heavy axinos followed by late-time decays in the early universe can augment the higgsino abundance. In this latter case, any remaining under-abundance can be filled by axions. In the case of higgsino dominance of the dark matter abundance, then we expect higgsinolike WIMPs to be detected by the next round of direct and indirect WIMP detection experiments. An axion signal could also be a viable possibility.

\section{Acknowledgments}

We thank Jenny List, Azar Mustafayev and Roman Nevzorov for discussions. This work was supported in part by the U.S. Department of Energy under grant Nos. DE-FG0204ER41305, DE-FG02-04ER41291 and DE-FG02-95ER40896.

\section{References}

[1] S. Dimopoulos and H. Georgi, Nucl. Phys. B 193 (1981) 150; N. Sakai, Z. Physik C 11 (1981) 153.

[2] H. Baer and X. Tata, Weak Scale Supersymmetry: From Superfields to Scattering Events, (Cambridge University Press, 2006).

[3] M. Drees, R. Godbole and P. Roy, Theory and Phenomenology of Sparticles, (World Scientific, 2004); P. Binetruy, Supersymmetry (Oxford University Press, 2006); S. P. Martin, hep-ph/9709356.

[4] S. Chatrchyan et al. (CMS collaboration), Phys. Rev. Lett. 107 (2011) 221804.

[5] G. Aad et al. (ATLAS collaboration), arXiv:1109.6572 (2011).

[6] See Steven Lowette, Talk at Rencontres de Moriond, La Thuile, Aosta Valley, Italy, March 3-10, 2012 for a very recent update.

[7] A. Arbey, M. Battaglia, A. Djouadi, F. Mahmoudi and J. Quevillon, arXiv:1112.3028.

[8] H. Baer, V. Barger and A. Mustafayev, arXiv:1202.4038.

[9] H. Baer, V. Barger and A. Mustafayev, arXiv:1112.3017.

[10] V. Kaplunovsky and J. Louis, Phys. Lett. B 306 (1993) 269.

[11] F. Gabbiani, E. Gabrielli, A. Masiero and L. Silvestrini, Nucl. Phys. B 477 (1996) 321 [arXiv:hep-ph/9604387].

[12] M. Dine, A. Kagan and S. Samuel, Phys. Lett. B 243 (1990) 250; A. Cohen, D. B. Kaplan and A. Nelson, Phys. Lett. B 388 (1996) 588;

[13] H. Baer, S. Kraml, A. Lessa, S. Sekmen and X. Tata, J. High Energy Phys. 1010 (2010) 018;

D. Feldman, G. Kane, E. Kuflik and R. Lu, Phys. Lett. B 704 (2011) 56.

[14] R. Arnowitt and P. Nath, Phys. Rev. D 46 (1992) 3981. 
[15] K. L. Chan, U. Chattopadhyay and P. Nath, Phys. Rev. D 58 (1998) 096004.

[16] N. Arkani-Hamed, talk at WG2 meeting, Oct. 31, 2012, CERN, Geneva.

[17] C. Brust, A. Katz, S. Lawrence and R. Sundrum, arXiv:1110.6670 [hep-ph].

[18] R. Essig, E. Izaguirre, J. Kaplan and J. G. Wacker, JHEP 1201 (2012) 074 [arXiv:1110.6443 [hep-ph]].

[19] M. Papucci, J. T. Ruderman and A. Weiler, arXiv:1110.6926 [hep-ph].

[20] S. F. King, M. Muhlleitner and R. Nevzorov, arXiv:1201.2671 [hep-ph].

[21] See e.g. H. Baer, S. Kraml, S. Sekmen and H. Summy, J. High Energy Phys. 0803 (2008) 056.

[22] S. Weinberg, Phys. Rev. Lett. 48 (1982) 1303, M. Khlopov and A. Linde, Phys. Lett. B 138 (1984) 265.

[23] M. Kawasaki, K. Kohri, T. Moroi and A. Yotsuyanagi, Phys. Rev. D 78 (2008) 065011.

[24] P. Moxhay and K. Yamamoto, Phys. Lett. B 151 (1985) 363; E. Chun and A. Lukas, Phys. Lett. B 357 (1995) 43.

[25] C. Cheung, G. Elor and L. J. Hall, Phys. Rev. D 85 (2012) 015008.

[26] K-Y. Choi, J. E. Kim, H. M. Lee and O. Seto, Phys. Rev. D 77 (2008) 123501.

[27] H. Baer, A. Lessa, S. Rajagopalan and W. Sreethawong, JCAP1106 (2011) 031.

[28] H. Baer, A. Lessa and W. Sreethawong, JCAP1201 (2012) 036.

[29] J. Ellis, K. Olive and Y. Santoso, Phys. Lett. B 539 (2002) 107; J. Ellis, T. Falk, K. Olive and Y. Santoso, Nucl. Phys. B 652 (2003) 259; H. Baer, A. Mustafayev, S. Profumo, A. Belyaev and X. Tata, Phys. Rev. D 71 (2005) 095008 and J. High Energy Phys. 0507 (2005) 065, and references therein.

[30] H. Baer, A. Belyaev, T. Krupovnickas and A. Mustafayev, JHEP 0406 (2004) 044.

[31] ISAJET, by H. Baer, F. Paige, S. Protopopescu and X. Tata, hep-ph/0312045.

[32] H. Baer, C. H. Chen, R. Munroe, F. Paige and X. Tata, Phys. Rev. D 51 (1995) 1046;

H. Baer, J. Ferrandis, S. Kraml and W. Porod, Phys. Rev. D 73 (2006) 015010.

[33] J. Hisano, H. Murayama, and T. Yanagida, Nucl. Phys. B402 (1993) 46. Y. Yamada, Z. Phys. C60 (1993) 83; J. L. Chkareuli and I. G. Gogoladze, Phys. Rev. D 58, 055011 (1998).

[34] S. Martin and M. Vaughn, Phys. Rev. D50, 2282 (1994).

[35] H. Haber and R. Hempfling, Phys. Rev. D 48 (1993) 4280.

[36] D. Pierce, J. Bagger, K. Matchev and R. Zhang, Nucl. Phys. B491, 3 (1997).

[37] Joint LEP 2 Supersymmetry Working Group, Combined LEP Chargino Results up to $208 \mathrm{GeV}$, http://lepsusy.web.cern.ch/lepsusy/www/inos_moriond01/charginos_pub.html.

[38] N. Arkani-Hamed and H. Murayama, Phys. Rev. D 56 (1997) 6733.

[39] H. Baer, C. Balazs, P. Mercadante, X. Tata and Y. Wang, Phys. Rev. D 63 (2001) 015011.

[40] G. Aad et al. (ATLAS Collaboration) arXiv:1202.1408 (2012); S. Kortner, talk at Rencontres de Moriond, La Thuile, Aosta Valley, March 3-10 (2012). 
[41] S. Chatrachyan et al. (CMS Collaboration) arXiv:1202.1488 (2012); M. Pieri, talk at Rencontres de Moriond, La Thuile, Aosta Valley, March 3-10 (2012).

[42] M. Carena, S. Gori, N. R. Shah and C. E. M. Wagner, arXiv:1112.3336 [hep-ph].

[43] H. Baer and M. Brhlik, Phys. Rev. D 55 (1997) 4463; H. Baer, M. Brhlik, D. Castano and X. Tata, Phys. Rev. D 58 (1998) 015007.

[44] D. Asner et al. (Heavy Flavor Averaging Group), arXiv:1010.1589 [hep-ex].

[45] G. W. Bennett et al. (Muon $g$ - 2 Collaboration), Phys. Rev. D 80 (2009) 052008.

[46] K. Cheung, C. -W. Chiang and J. Song, JHEP 0604 (2006) 047.

[47] H. Baer, V. Barger and P. Huang, JHEP 1111 (2011) 031.

[48] W. Beenakker, R. Hopker and M. Spira, hep-ph/9611232.

[49] H. Baer, V. Barger, A. Lessa and X. Tata, J. High Energy Phys. 1006 (2010) 102 and arXiv:1112.3044.

[50] H. Baer, X. Tata and J. Woodside, Phys. Rev. D 45 (1992) 142; H. Baer, C. H. Chen, F. Paige and X. Tata, Phys. Rev. D 52 (1995) 2746 and Phys. Rev. D 53 (1996) 6241, H. Baer, C. H. Chen, M. Drees, F. Paige and X. Tata, Phys. Rev. D 59 (1999) 055014 H. Baer, C. Balázs, A. Belyaev, T. Krupovnickas and X. Tata, J. High Energy Phys. 0306 (2003) 054; H. Baer, V. Barger, A. Lessa and X. Tata, J. High Energy Phys. 0909 (2009) 063; see also, S. Abdullin and F. Charles, Nucl. Phys. B 547 (1999) 60; S. Abdullin et al. (CMS Collaboration), J. Phys. G 28 (2002) 469 [hep-ph/9806366]; B. Allanach, J. Hetherington, A. Parker and B. Webber, J. High Energy Phys. 08 (2000) 017.

[51] U. Chattopadhyay, A. Datta, A. Datta, A. Datta and D. P. Roy, Phys. Lett. B 493 (2000) 127; P. G. Mercadante, J. K. Mizukoshi and X. Tata, Phys. Rev. D 72 (2005) 035009;

S. P. Das, A. Datta, M. Guchait, M. Maity and S. Mukherji, Eur. Phys. J. C 54 (2008) 645; R. Kadala, P. G. Mercadante, J. K. Mizukoshi and X. Tata, Eur. Phys. J. C 56 (2008) 511.

[52] A. Marzin, talk at Rencontres de Moriond, La Thuile, Aosta Valley, March 3-10 (2012).

[53] H. Baer, A. Belyaev, T. Krupovnickas and X. Tata, J. High Energy Phys. 0402 (2004) 007. H. Baer, T. Krupovnickas and X. Tata, J. High Energy Phys. 0406 (2004) 061.

[54] T. Moroi and L. Randall, Nucl. Phys. B 570 (2000) 455; G. Gelmini and P. Gondolo, Phys. Rev. D 74 (2006) 023510; G. Gelmini, P. Gondolo, A. Soldatenko and C. Yaguna, Phys. Rev. D 74 (2006) 083514; G. Gelmini, P. Gondolo, A. Soldatenko and C. Yaguna, Phys. Rev. D 76 (2007) 015010; B. Acharya, K. Bobkov, G. Kane, P. Kumar and J. Shao, Phys. Rev. D 76 (2007) 126010 and Phys. Rev. D 78 (2008) 065038; B. Acharya, P. Kumar, K. Bobkov, G. Kane, J. Shao and S. Watson, J. High Energy Phys. 0806 (2008) 064.

[55] H. Baer, C. Balazs, A. Belyaev and J. O'Farrill, JCAP 0309 (2003) 007.

[56] E. Aprile et al. (XENON100 Collaboration) Phys. Rev. Lett. 107 (2011) 131302.

[57] R. Abbasi et al. (IceCube collaboration), arXiv:1112.1840.

[58] E. Behnke, J. Behnke, S. J. Brice, D. Broemmelsiek, J. I. Collar, P. S. Cooper, M. Crisler and C. E. Dahl et al., Two Liter $\mathrm{CF}_{3} \mathrm{I}$ Bubble Chamber," Phys. Rev. Lett. 106 (2011) 021303; W. H. Lippincott (COUPP collaboration), talk at TAUP conference, Munich, Germany, September 7, 2011.

[59] M. Ackermann et al. (Fermi Collaboration), Phys. Rev. Lett. 107 (2011) 241302; A. Geringer-Sameth and S. M. Koushiappas, Phys. Rev. Lett. 107 (2011) 241303. 